\title{
Flora y vegetación de la Reserva Nacional Lago Peñuelas, Reserva de la Biósfera, Región de Valparaíso, Chile
}

\author{
Flora and vegetation of the National Reserve Lago Peñuelas, \\ Biosphere Reserve, Region of Valparaiso, Chile
Enrique Hauensteina*, Andrés Muñoz-Pedreros ${ }^{\mathrm{a}}$, José Yánez ${ }^{\mathrm{b}, \mathrm{c}}$, Pamela Sánchez ${ }^{\mathrm{a}}$, Patricia Möller ${ }^{\mathrm{b}}$, Basilio Guiñez $^{\mathrm{a}}$, Claudia Gil ${ }^{\mathrm{b}}$

\begin{abstract}
*Autor de correspondencia: aniversidad Católica de Temuco, Facultad de Recursos Naturales, Escuela de Ciencias Ambientales, casilla 15-D, Temuco, Chile, tel.: 45-205467, ehauen@uct.cl

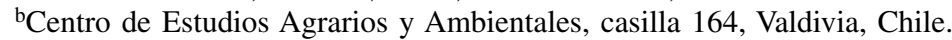

${ }^{\mathrm{c} M u s e o}$ Nacional de Historia Natural, casilla 787, Santiago, Chile.
\end{abstract}

\begin{abstract}
SUMMARY
The National Reserve Lago Peñuelas belongs to the National System of Protected Wild Areas, located in the Region of Valparaiso, Chile. This unit, together with the National Park La Campana, constitutes a Biosphere Reserve. The flora and vegetation of the National Reserve was studied. The study registered 163 species of vascular plants, of which 48 are new records. Considering the studies and previous reports made in situ, the floristic richness reaches 337 species, of which $72 \%$ are native; eight of them are pteridophytes, two are gimnospermae, 229 are dicotyledoneae and 98 are monocotyledoneae. This represents a high floristic diversity. Nevertheless, the high proportion of aloctonous plants indicates a relative high degree of anthropic disturbance. Fourteen species have conservation problems (two of them are threatened, eleven are vulnerable and one is data deficient). The total phytosociology of the place reports the presence of 20 plant communities, ten of which are herbaceous, seven scrub and three tree communities; of these, 12 have already been described in previous works, adding the present study eight new associations for the place. It is proposed to keep as restricted areas the sector of Los Lirios, which contains most of the threatened species, and the sector of Vega del Alamo, because of its valuable condition as a relict of the hygrophilous forest present.
\end{abstract}

Key words: biodiversity, conservation, phytosociology, Peñuelas Lake, Chile.

\section{RESUMEN}

La Reserva Nacional Lago Peñuelas es una unidad del Sistema Nacional de Áreas Silvestres Protegidas del Estado ubicada en la Región de Valparaíso, Chile. Esta unidad, junto al Parque Nacional La Campana, constituye una Reserva de la Biósfera. Se estudió la flora y vegetación de esta Reserva Nacional. El estudio registró 163 especies de plantas vasculares, de las cuales 48 son nuevos registros a la documentación previa. Al considerar los estudios y reportes realizados en el lugar, la riqueza florística alcanza a 337 especies, de las cuales $72 \%$ es de origen nativo, distribuidas en ocho pteridófitos, dos gimnospermas, 229 dicotiledóneas y 98 monocotiledóneas, lo que representa una alta riqueza florística. Sin embargo, la mayor proporción de plantas alóctonas indica también un fuerte grado de perturbación antrópica. Catorce especies presentan problemas de conservación (dos en peligro de extinción, 11 vulnerables y una insuficientemente conocida). La fitosociología total del lugar reporta la presencia de 20 comunidades vegetales, de las cuales 10 son herbáceas, siete de matorral y tres arbóreas; de éstas, 12 ya habían sido descritas en trabajos anteriores, agregando el presente estudio ocho asociaciones nuevas para el lugar. Se propone mantener como áreas restringidas de esta reserva, el sector Los Lirios, que contiene la mayor parte de las especies amenazadas, y el sector Vega del Álamo por la valiosa condición de relicto del bosque higrófilo allí presente.

Palabras clave: biodiversidad, conservación, fitosociología, lago Peñuelas.

\section{INTRODUCCIÓN}

El concepto de área protegida es amplio y su definición es discutida en la literatura (CONAF 1992, UICN 1994). Si bien la amplitud de este concepto puede diferir, el objetivo es muy similar, la conservación de la naturaleza.

En Chile existe el Sistema Nacional de Áreas Silvestres Protegidas del Estado (SNASPE), el cual comprende tres categorías: Parques Nacionales (PN), Reservas Nacionales (RN) y Monumentos Naturales (MN). Actualmente comprende 95 unidades, las que en total cubren el 19\% del territorio nacional. El SNASPE se ha constituido en un pilar fundamental para salvaguardar no sólo parte importante del patrimonio natural de Chile, sino que también para proteger y valorizar nuestro acervo cultural, particularmente el que se encuentra inserto en las áreas que conforman dicho 
sistema (Oltremari 2002). En la Región de Valparaíso se encuentra la Reserva Nacional Lago Peñuelas, declarada en conjunto con el Parque Nacional La Campana, Reserva de la Biósfera, debido a que posee una alta diversidad biológica y ecosistémica, representativa de ambientes mediterráneos característicos de esa región (Weber 1986, CONAF 1994, Elórtegui y Moreira-Muñoz 2002).

La zona central de Chile, denominada también zona mesomórfica o mediterránea (Pisano 1956), se extiende aproximadamente entre los $32^{\circ}$ y $37^{\circ} \mathrm{S}$. Posee un clima de tipo mediterráneo, que Köppen (1931) clasifica como "clima templado-cálido con humedad suficiente (Cfa, $\mathrm{Csb})$ " y en la subdivisión de "lluvias invernales y estación seca prolongada", caracterizada por periodos de lluvia regulares durante el invierno y una estación seca bien marcada, que puede extenderse entre seis y ocho meses, existiendo una correspondencia con otras áreas mediterráneas mundiales como California y Europa en el Hemisferio Norte, y Australia y sur de África en el Hemisferio Sur (Grau 1992, Arroyo et al. 1995). Estas características climáticas condicionan que la vegetación de estas regiones posea características adaptativas especiales, como son, entre otras, la presencia de hojas esclerófilas, de lignotúber y de una gran capacidad de economía hídrica (Money y Kumerow 1971, Araya y Ávila 1981, Ávila et al. 1981). De acuerdo a Marticorena et al. (1995) la zona central de Chile es un foco de concentración de endemismos y de una alta riqueza y diversidad florística. Mittermeier et al. (1998) señalan la presencia de 1.800 especies de plantas endémicas para esta área, lo que la ha llevado a ser considerada una de las 25 áreas hotspots mundiales, que requieren prioridad de protección (Myers et al. 2000).

En este contexto, los estudios de flora y vegetación son fundamentales como elementos de base para el desarrollo de propuestas de conservación o manejo de especies y ecosistemas, o para definir áreas prioritarias (Cavieres et al. 2001, Teillier et al. 2005). Son destacables de mencionar, en zonas aledañas al área de estudio, los trabajos de Looser (1944), Villaseñor (1980, 1986), Villaseñor y Serey (1980-1981), Balduzi et al. (1981, 1982), Palma et al. (1978), Ramírez et al. (1987), Zöllner et al. (1995), Arroyo et al. (2000, 2005), Elórtegui y Moreira (2002); y específicamente en la misma Reserva Nacional, de ICSA (1980) y CONAF $(1986,1994)$. Es en estos tres últimos trabajos donde se informa de un total de 283 especies registradas y de 12 unidades de vegetación, al mismo tiempo que se destaca la presencia de especies bulbosas y de flores llamativas en algunos sectores de la Reserva, como en el sector Los Lirios.

El presente trabajo pretende complementar la información florística y vegetacional existente de la Reserva Nacional Lago Peñuelas, con el objeto de que la información entregada permita reforzar su importancia como sitio relevante de biodiversidad y a la vez pueda ser utilizada para mejorar aspectos de su conservación y manejo.

\section{MÉTODOS}

Área de estudio. La RN Lago Peñuelas fue creada el año 1952 y posee una superficie de 9.260 ha. El área de estudio comprendió las 2.473 ha con cubierta vegetal natural, estando la superficie restante cubierta por vegetación exótica, dominada por plantaciones de pino insigne (Pinus radiata D. Don) y eucalipto (Eucalyptus globulus Labill.), y otra parte correponde al espejo de agua del lago Peñuelas. Se ubica entre las coordenadas $33^{\circ} 07^{\prime} \mathrm{S}$ y $71^{\circ} 24^{\prime} \mathrm{O}$, a $30 \mathrm{~km}$ al sureste de la ciudad de Valparaíso. Se sitúa en una cuenca cerrada con una extensión de $17 \mathrm{~km}$ y altitudes que van desde los $337 \mathrm{~m}$ s.n.m. en el espejo de agua del lago Peñuelas, hasta los 613 m s.n.m. (cerro Mutilemu). El clima del área es de tipo mediterráneo templado, con humedad suficiente, lluvias invernales y estación seca prolongada. Presenta temperatura media anual de $13,5^{\circ} \mathrm{C}$, la máxima media es de $17,1^{\circ} \mathrm{C}$ y la mínima media es de $9,4^{\circ} \mathrm{C}$; rara vez la temperatura baja de $0^{\circ} \mathrm{C}$, presentándose heladas desde mayo a septiembre. Las precipitaciones son estacionales ( $656 \mathrm{~mm} / \mathrm{año})$, comenzando a fines de mayo hasta agosto, pasando a un verano marcadamente seco de seis a ocho meses (Di Castri y Hajek 1976, Luebert y Pliscoff 2006).

Respecto de la flora, en febrero y noviembre de 2001 se realizaron recolecciones intensivas y se aplicaron 27 relevamientos fitosociológicos en nueve estaciones de muestreo (cuadro 1). La determinación y origen geográfico de cada especie se basó en Muñoz (1966), Navas (1973, 1976, 1979), Hoffmann (1978, 1991), Marticorena y Quezada (1985), Matthei (1995), Hoffmann et al. (1998) y Marticorena y Rodríguez (1995, 2001, 2003, 2005). Para la actualización de los nombres científicos y sus abreviaturas, se utilizó el índice internacional de nombres de plantas (IPNI 2008). Las formas de vida se determinaron de acuerdo al esquema propuesto por Ellenberg y Mueller-Dombois (1966). El estado de conservación se determinó considerando las propuestas de Benoit (1989), actualizadas en reunión de expertos en septiembre de 1997 (Baeza et al. 1998, Belmonte et al. 1998, Ravenna et al. 1998), y de Novoa et al. (2006) para las Orchidaceae. El grado de perturbación antrópica del lugar se determinó sobre la base de lo propuesto por Hauenstein et al. (1988) y la escala de evaluación de González (2000), que consideran el origen fitogeográfico, es decir, la relación entre las especies nativas e introducidas, y las formas de vida (formas biológicas de Raunkiaer) como medidas de esta forma de perturbación. Como resultado de lo anterior se obtuvo un inventario de la flora del lugar (anexo 1) que contiene todos los elementos antes mencionados y en el cual se incorporaron con fines comparativos, además de las especies registradas en este estudio, aquellas mencionadas en otros trabajos realizados en la RN Lago Peñuelas o que mencionan especies recolectadas en dicho lugar. Así, las especies registradas en este estudio se identifican con el número 1, con el 2 ICSA (1980), con el 3 CONAF (1986), 
con el 4 CONAF (1994), con el 5 Zöllner et al. (1995) y con el 6 Novoa et al. (2006). Las plantas recolectadas quedaron depositadas en el Herbario de la Escuela de Ciencias Ambientales (UCT- Herbario no oficial), Facultad de Recursos Naturales, de la Universidad Católica de Temuco; también se preparó una colección de plantas herborizadas con 127 especies, de acuerdo a las técnicas tradicionales para este efecto (Hauenstein y Parada 1975), el cual quedó como referencia en dependencias de CONAF Región de Valparaíso.

En relación a la vegetación, para la determinación de las unidades de vegetación se tomó como base la cartografía producida por CONAF-CONAMA-BIRF (1999) y cartografía específica entregada por CONAF Región de Valparaíso. La definición y nomenclatura de éstas se hizo de acuerdo a la propuesta de Gajardo (1995). Posteriormente se verificaron en terreno las unidades vegetacionales señaladas en la cartografía. Se aplicaron relevamientos fitosociológicos de acuerdo a la metodología fitosociológica europea (BraunBlanquet 1964, 1979). La superficie de cada uno de los inventarios fue de $4 \mathrm{~m}^{2}$ para la vegetación herbácea, de $25 \mathrm{~m}^{2}$ para la arbustiva y de $100 \mathrm{~m}^{2}$ para la boscosa, en superficies superiores al área mínima (Steubing et al. 2002). Se consideró también la vegetación acuática y palustre de las riberas del lago Peñuelas. Las tablas fitosociológicas se procesaron de acuerdo a la metodología propuesta por Braun-Blanquet (1964), explicitada en Mueller-Dombois y Ellenberg (1974) y Ramírez y Westermeier (1976). En ellas los valores de cobertura de cada especie en cada inventario se estimaron visualmente y se expresaron directamente en porcentaje, lo que permitió un trabajo más expedito en terreno y para la obtención del valor de importancia de las especies.

Para cada especie se determinó el valor de importancia, el cual se obtiene de la suma de las frecuencias y coberturas relativas $(\mathrm{Fr}+\mathrm{Cr})$, de acuerdo a Wikum y Shanholtzer (1978), lo que da cuenta de la abundancia e importancia de cada especie en el lugar estudiado. Para esto, en una segunda tabla, se consideraron los valores de cobertura en porcentaje y a los signos + y $\mathrm{r}$ se les asigna valor 1 . Con este dato se procedió a anotar en el margen derecho de dicha tabla y después de la columna correspondiente al último inventario, la frecuencia (constancia) de cada especie, o sea el número de inventarios en que ella estuvo presente. Dicha frecuencia se indicó en una columna en números absolutos $(\mathrm{F})$, y en otra, en términos de frecuencia relativa (Fr), que indicó el porcentaje de la frecuencia de cada especie, haciendo equivalente al $100 \%$ la sumatoria de las frecuencias (F). A partir de la tercera columna se anotaron los valores correspondientes a la cobertura total por especies (C) y su cobertura relativa (Cr), usando como $100 \%$ la sumatoria de las coberturas (C).

\section{RESULTADOS}

Flora. La flora vascular registrada en el presente estudio (anexo 1) comprendió 163 especies de plantas vasculares, distribuidas en cuatro pteridófitos (2,5\%), dos gimnospermas (1,2\%), 107 dicotiledóneas $(65,6 \%)$ y 50 monocotiledóneas $(30,7 \%)$. El presente estudio incorporó 48 nuevas especies a los catálogos existentes de la flora del lugar (ICSA 1980, CONAF 1986, 1994), con los cuales se reúnen 337 especies. El espectro biológico (anexo 1) estuvo representado por 51 terófitos $(31,3 \%)$, que comprenden a plantas anuales y bienales, 34 hemicriptófitos (20,9\%), 32 criptófitos $(19,6 \%)$, que incluyen a hidrófitos y geófitos, 25 fanerófitos $(15,3 \%), 19$ nanofanerófitos $(11,7 \%)$ y sólo dos caméfitos $(1,2 \%)$. El origen fitogeográfico (anexo 1) indicó que $64,4 \%$ de las especies (105 sp.) eran nativas y $35,6 \%$ introducidas (58 sp.).

El estado de conservación de las especies (anexo 1, cuadro 2), considerando el total de especies mencionadas para el lugar, mostró que la mayoría de ellas no presentó problemas, determinándose sólo 14 taxa $(4,1 \%)$ con problemas de conservación. De ellas, dos se encuentran en la categoría de "en peligro" de extinción, como es el caso de Beilschmiedia miersii (belloto del norte) y Calydorea

Cuadro 1. Caracterización y localización de nueve estaciones de muestreo en la Reserva Nacional Lago Peñuelas, 2001 (coordenadas en UTM).

Characterization and location of nine stations of sampling in the National Reserve Lago Peñuelas, 2001 (coordinates in UTM).

\begin{tabular}{|c|c|c|c|}
\hline Estación & Sector & Coordenadas & Formación vegetal \\
\hline 1 & La Engorda. Camino las Palmas & 274.670 E $6.326 .009 \mathrm{~N}$ & Estepa de Acacia caven \\
\hline 2 & La Vega del Álamo. Puerta de entrada & 275.381 E $6.326 .052 \mathrm{~N}$ & Bosque esclerófilo \\
\hline 3 & La Vega del Álamo. Abrevadero & 275.942 E $6.326 .648 \mathrm{~N}$ & Bosque higrófilo \\
\hline 4 & Este del lago. Puente eucaliptos & 270.882 E $6.327 .190 \mathrm{~N}$ & Totoral y pradera húmeda \\
\hline 5 & Noreste del lago. La Chicuta & 268.009 E $6.327 .880 \mathrm{~N}$ & Matorral mixto de Acacia y Baccharis \\
\hline 6 & La Engorda. La Pérgola & 272.002 E $6.324 .869 \mathrm{~N}$ & Estepa de Acacia caven \\
\hline 7 & Los Lirios. Junto al estero & 268.553 E $6.325 .122 \mathrm{~N}$ & Matorral mixto de Baccharis y Puya \\
\hline 8 & La Engorda. Entrada & 271.092 E $6.325 .425 \mathrm{~N}$ & Estepa de Acacia caven \\
\hline 9 & La Engorda. Junto al estero & 272.810 E $6.324 .215 \mathrm{~N}$ & Bosquete esclerófilo \\
\hline
\end{tabular}


xiphioides, 11 en la categoría de "vulnerable", correspondiendo principalmente a especies bulbosas (geófitas) como Herbertia lahue, Leucocoryne ixioides, L. violacescens y Hippeastrum bicolor. Sólo una especie se encuentra en la categoría de "insuficientemente conocida", es el caso de Blechnum chilense del que se registró sólo un ejemplar en el sector El Abrevadero.

Cuadro 2. Plantas vasculares con problemas de conservación en la Reserva Nacional Lago Peñuelas (EC = estado de conservación, $\mathrm{P}=$ en peligro, $\mathrm{V}=$ vulnerable, $\mathrm{IC}=$ insuficientemente conocida).

Vascular plants with conservation problems of the National Reserve Lago Peñuelas $(\mathrm{P}=$ threatened, $\mathrm{V}=$ vulnerable, $\mathrm{IC}=$ data deficient).

\begin{tabular}{lcl}
\hline Especie & EC & Fuente bibliográfica \\
\hline Alstroemeria hookeri & V & Ravenna et al. (1998) \\
Beilschmiedia miersii & P & Benoit (1989) \\
Blechnum chilense & IC & Baeza et al. (1998) \\
Blepharocalyx cruckshanksii & V & Benoit (1989) \\
Calydorea xyphioides & P & Ravenna et al. (1998) \\
Chloraea heteroglossa & V & Ravenna et al. (1998), Novoa \\
& & et al. (2006) \\
C. disoides & V & Ravenna et al. (1998), Novoa \\
& & et al. (2006) \\
Conanthera trimaculata & $\mathrm{V}$ & Benoit (1989) \\
Herbertia lahue & $\mathrm{V}$ & Benoit (1989) \\
Leucocoryne ixioides & $\mathrm{V}$ & Benoit (1989) \\
L. violacescens & $\mathrm{V}$ & Benoit (1989) \\
Neoporteria curvispina & $\mathrm{V}$ & Benoit (1989), Belmonte et \\
Phycella bicolor & & al. (1998) \\
Puya chilensis & $\mathrm{V}$ & Benoit (1989) \\
\hline
\end{tabular}

Vegetación. La fitosociología del lugar indica que la vegetación leñosa (cuadro 3) está integrada por matorral abierto de espino, matorral esclerófilo mixto, bosque esclerófilo y bosque higrófilo. La herbácea (cuadro 4), por praderas secas, praderas húmedas, totoral, ciperal y comunidad de acuáticas, las que se caracterizan a continuación:

a) Matorral abierto de espino. Representada por los inventarios 1 a 5 (cuadro 3). En esta formación domina el espino (Acacia caven = Vachellia caven) junto con el maitén (Maytenus boaria), cuyas coberturas promedio fueron de $50 \%$ y menos de $10 \%$, respectivamente, por lo que el estrato arbóreo era bastante ralo. Se caracterizó por la presencia de un rico estrato herbáceo, en el que destacaron Agrostis capillaris, Leontodon saxatilis, Avena barbata, Bromus hordeaceus, Briza minor, B. maxima y Rhodophiala advena, existiendo un claro predominio de gramíneas forrajeras. b) Matorral esclerófilo mixto. Representado por los inventarios 6 a 8 (cuadro 3). En éste se entremezclaron especies de la estepa de acacia con elementos del bosque esclerófilo, tales como Quillaja saponaria, Schinus latifolia, Cryptocarya alba, junto a especies arbustivas, como Schinus polygama, Baccharis linearis, Berberis actinacantha, Eryngium paniculatum y Satureja gilliesii. Esta comunidad puede ser caracterizada como un matorral abierto de espino y romerillo (Baccharis linearis). Las especies leñosas dominantes fueron Acacia caven con cerca de $20 \%$ de cobertura y B. linearis con un $10 \%$; le siguieron Maytenus boaria, Quillaja saponaria y Schinus latifolia; con menor cobertura se encontraron Peumus boldus, Lithraea caustica, Escallonia pulverulenta y la trepadora Muehlenbeckia hastulata. El estrato herbáceo, cuya cobertura promedio fue de $90 \%$, estuvo representado por las especies Agrostis capillaris, Avena barbata, Poa annua, Festuca sp., Bromus hordeaceus, Briza minor y Leontodon saxatilis.

c) Bosque esclerófilo. Representado por los inventarios 9 a 15 (cuadro 3). Esta es una formación leñosa típica de la región. Esta comunidad corresponde al Peumo-Cryptocaryetum albae (Oberdorfer 1960). Es posible encontrar algunas variantes de esta comunidad con especies del matorral esclerófilo. En ella la cobertura promedio del estrato arbóreo fue de $70 \%$, la de arbustos $35 \%$ y la herbácea de $25 \%$. Destacaron en el estrato arbóreo, Cryptocarya alba, Peumus boldus y Quillaja saponaria; en el arbustivo, Retanilla trinervia, Schinus latifolia y Azara dentata, y en el herbáceo Alonsoa meridionalis, Conyza floribunda, Anagallis arvensis y Marrubium vulgare. En el inventario 15 se apreció la fuerte presencia de Pinus radiata, que alcanzó una cobertura de 50\%; lo que muestra en algunas áreas la fuerte participación de elementos alóctonos.

d) Bosque higrófilo. Representado por los inventarios 16 y 17 (cuadro 3). Comprendió pequeños remanentes boscosos asociados a cursos permanentes de agua y vertientes, como ocurrió en el sector Vega del Álamo. Está compuesto por especies higrófilas como Drimys winteri, Myrceugenia obtusa, Blapharocalyx cruckschanksii y Luma chequen, y algunas trepadoras como Lardizabala biternata y Cissus striata. Corresponde a la asociación Blepharocalyo-Myrceugenietum exsuccae, hualve o bosque pantanoso de mirtáceas de la zona centro-sur de Chile (Ramírez et al. 1996). Estos bosques se incluyen en la clase fitosociológica WinteroNothofagetea de Oberdorfer (1960). Las especies leñosas más importantes de este bosque higrófilo fueron: Drimys winteri, Myrceugenia obtusa, Maytenus boaria y Escallonia revoluta, junto a algunas trepadoras como Cissus striata y Lardizabala biternata. El estrato herbáceo fue bastante pobre, debido probablemente a lo cerrado del dosel que impide la penetración de luz, y estuvo representado por Uncinia trichocarpa y helechos como Blechnum hastatum y B. chilense. 
Cuadro 3. Estructura florística de la vegetación leñosa de la Reserva Nacional Lago Peñuelas $(\mathrm{A}=$ matorral de acacia, $\mathrm{B}=$ matorral esclerófilo mixto, $\mathrm{C}=$ bosque esclerófilo, $\mathrm{D}=$ bosque higrófilo; $\mathrm{VI}=$ valor de importancia; valores numéricos bajo los inventarios $=$ porcentajes de cobertura, $+=$ cobertura $<5 \%$ ).

Floristic structure of the wood vegetation of National Reserve Lago Peñuelas (A = acacia scrub, B = mixed sclerophilous scrub, $\mathrm{C}=$ sclerophilous forest, $\mathrm{D}=$ hygrophilous forest $\mathrm{VI}=$ value of importance; numerical values under the inventories $=$ percentages of coverage, $+=$ coverage $<5 \%)$.

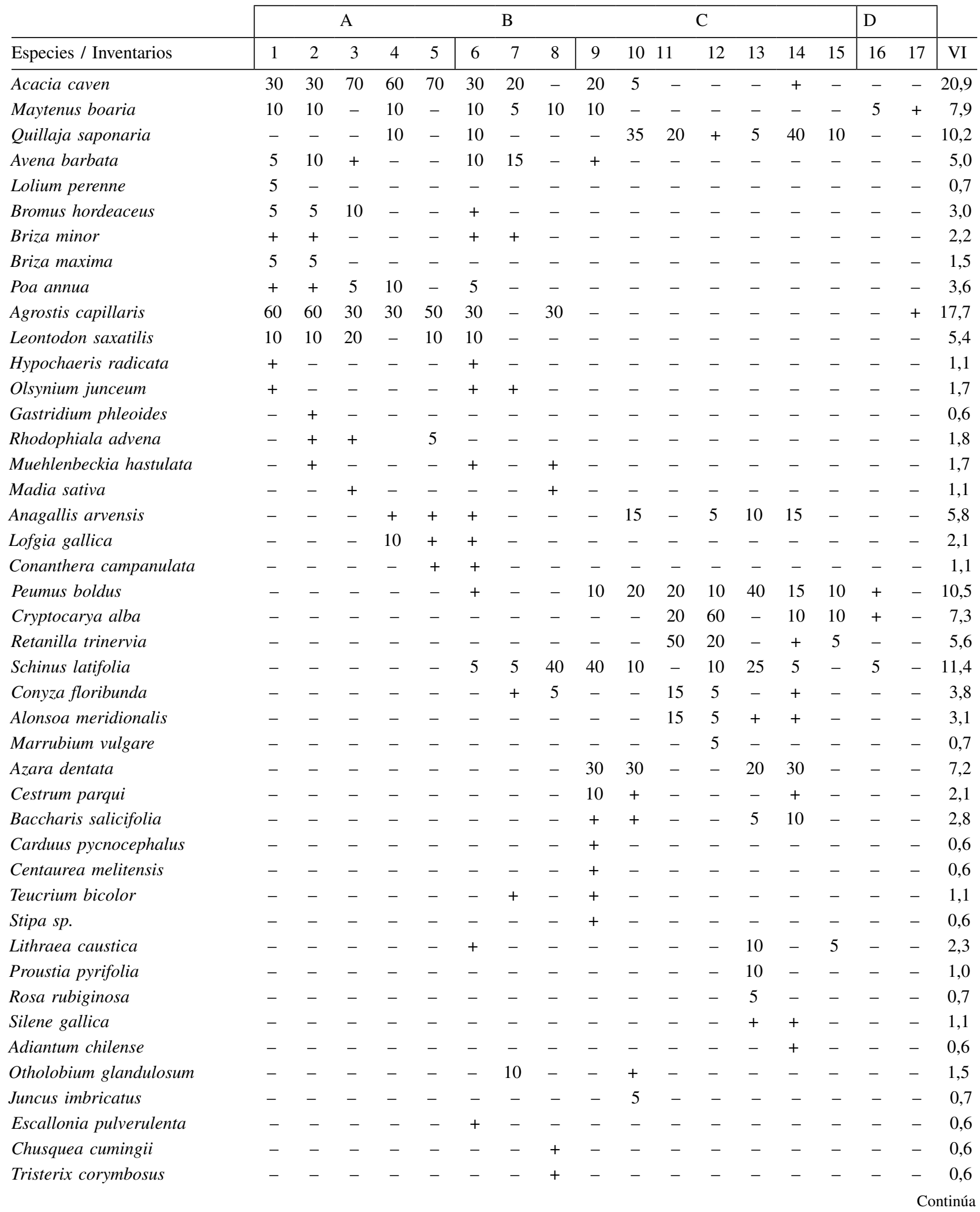


BOSQUE 30(3): 159-179, 2009

Flora y vegetación RN Lago Peñuelas, Chile

\begin{tabular}{|c|c|c|c|c|c|c|c|c|c|c|c|c|c|c|c|c|c|c|}
\hline \multirow{2}{*}{$\begin{array}{l}\text { Continuación del Cuadro } 3 \\
\text { Especies / Inventarios }\end{array}$} & \multicolumn{6}{|c|}{ A } & \multicolumn{2}{|l|}{ B } & \multicolumn{6}{|c|}{$\mathrm{C}$} & \multicolumn{3}{|c|}{$\mathrm{D}$} & \multirow[b]{2}{*}{ VI } \\
\hline & 1 & 2 & 3 & 4 & 5 & 6 & 7 & 8 & 9 & 10 & 11 & 12 & 13 & 14 & 15 & 16 & 17 & \\
\hline Eryngium paniculatum & - & - & - & - & - & - & 5 & 5 & - & - & - & - & - & - & - & - & - & 1,5 \\
\hline Rumex conglomeratus & - & - & - & - & - & - & + & + & - & - & - & - & - & - & - & - & - & 1,1 \\
\hline Eragrostis virescens & - & - & - & - & - & - & - & 5 & - & - & - & - & - & - & - & - & - & 0,7 \\
\hline Festuca sp. & - & - & - & - & - & 20 & - & - & - & - & - & - & - & - & - & - & - & 1,4 \\
\hline Salix babylonica & - & - & - & - & - & + & - & - & - & - & - & - & - & - & - & - & - & 0,6 \\
\hline Acacia melanoxylon & - & - & - & - & - & + & - & - & - & - & - & - & - & - & - & - & - & 0,6 \\
\hline Baccharis linearis & - & - & - & - & - & 20 & 5 & - & - & - & - & - & - & - & - & - & - & 2,2 \\
\hline Schinus polygama & - & - & - & - & - & - & 5 & - & - & - & - & - & - & - & - & - & - & 0,7 \\
\hline Satureja gilliesii & - & - & - & - & - & - & 30 & - & - & - & - & - & - & - & - & - & - & 2,1 \\
\hline Berberis actinacantha & - & - & - & - & - & - & 5 & - & - & - & - & - & - & - & - & - & - & 0,7 \\
\hline Oenothera affinis & - & - & - & - & - & - & + & - & - & - & - & - & - & - & - & - & - & 0,6 \\
\hline Aristeguietia salvia & - & - & - & - & - & - & - & - & - & - & - & - & - & - & 5 & - & - & 0,7 \\
\hline Podanthus mitiqui & - & - & - & - & - & - & - & - & - & - & - & - & - & - & 10 & - & - & 1,0 \\
\hline Pinus radiata & - & - & - & - & - & - & - & - & - & - & - & - & - & - & 50 & - & - & 2,9 \\
\hline Senna candolleana & - & - & - & - & - & - & - & - & - & - & - & - & - & - & + & - & - & 0,6 \\
\hline Drimys winteri & - & - & - & - & - & - & - & - & - & - & - & - & - & - & & 50 & 40 & 5,2 \\
\hline Blepharocalyx cruckshanksii & - & - & - & - & - & - & - & - & - & - & - & - & - & - & - & 5 & 5 & 1,5 \\
\hline Myrceugenia obtusa & - & - & - & - & - & - & - & - & - & - & - & - & - & - & - & 20 & 30 & 3,4 \\
\hline Escallonia revoluta & - & - & - & - & - & - & - & - & - & - & - & - & - & - & - & 10 & - & 1,0 \\
\hline Luma chequen & - & - & - & - & - & - & - & - & - & - & - & - & - & - & - & + & - & 0,6 \\
\hline Aristotelia chilensis & - & - & - & - & - & - & - & - & - & - & - & - & - & - & - & + & - & 0,6 \\
\hline Rhaphithamnus spinosus & - & - & - & - & - & - & - & - & - & - & - & - & - & - & - & - & + & 0,6 \\
\hline Rubus ulmifolius & - & - & - & - & - & - & - & - & - & - & - & - & - & - & - & + & - & 0,6 \\
\hline Cissus striata & - & - & - & - & - & - & - & - & - & - & - & - & - & - & - & + & + & 1,1 \\
\hline Lardizabala biternata & - & - & - & - & - & - & - & - & - & - & - & - & - & - & - & + & + & 1,1 \\
\hline Solanum furcatum & - & - & - & - & - & - & - & - & - & - & - & - & - & - & - & + & + & 1,1 \\
\hline Uncinia trichocarpa & - & - & - & - & - & - & - & - & - & - & - & - & - & - & - & + & + & 1,1 \\
\hline Blechnum hastatum & - & - & - & - & - & - & - & - & - & - & - & - & - & - & - & + & + & 1,1 \\
\hline Total especies & 12 & 12 & 8 & 7 & 7 & 22 & 16 & 12 & 12 & 10 & 6 & 9 & 11 & 14 & 10 & 16 & 11 & - \\
\hline
\end{tabular}

e) Praderas secas. Representadas por los inventarios 1 a 3 (cuadro 4). Los principales componentes florísticos de estas praderas fueron Phyla canescens, Bromus hordeaceus Agrostis capillaris, Hypochaeris radicata, Gamochaeta coarctata, Leontodon saxatilis, Rumex acetosella y Avena barbata; corresponde a la comunidad Bromo-Lolietum (Oberdorfer 1960). Se ubicaron en el sector nororiente del lago.

f) Praderas húmedas. Representadas por los inventarios 4 a 7 (cuadro 4). Existen dos tipos, en las que, además de algunas de las especies de la comunidad anterior, destacaron en una (inventarios 4 y 5) la presencia de Mentha pulegium y juncos (Juncus acutus, J. cyperoides, J. pallescens). Esta comunidad correspondió a la pradera húmeda de junquillo (Juncetum acutii (ass. nov.)). En la otra (inventarios 6 y 7) destacaron Ludwigia peploides, Cotula coronopifolia, Distichlis spicata y Paspalum dilatatum, correspondiendo al Polygono-Ludwigietum peploidis (Steubing et al. 1980). Se ubican en las planicies norte y sur próximas al lago, aunque el Polygono-Ludwigietum ocupa la franja más cercana al agua. g) Totoral y ciperal. Representado por los inventarios 8 y 9 (cuadro 4). Estas comunidades se ubican de preferencia en el canal que desemboca al lago por el lado oriental. La primera de ellas, ubicada más próxima al agua, correspondió al Scirpetum californiae (Ramírez y Añazco 1982) (inventario 9), ya que su principal componente fue la totora (Schoenoplectus californicus), que estaba acompañada por especies como Ludwigia peploides y Polygonum hydropiperoides. La segunda comunidad, que se ubica en una franja continua un tanto más alejada del agua (inventario 8), correspondió al Loto-Cyperetum eragrostidae (San Martín et al. 1993), cuyas especies principales fueron Cyperus eragrostis y Carex excelsa (cortaderas), acompañadas por Juncus pallescens y J. acutus; es una asociación pantanosa y perenne, típica en depresiones y riberas de cursos de agua, asociada al totoral o a bordes del bosque pantanoso de temo (Blepharocalyx cruckshanksii (Hooker et Arnott) Niedenzu) y pitra (Myrceugenia exsucca (DC) Berg) (Hauenstein et al. 2002, San Martín et al. 2002). 
Cuadro 4. Estructura florística de la vegetacion herbácea de la Reserva Nacional Lago Peñuelas $(\mathrm{A}=$ pradera seca, $\mathrm{B}=$ pradera húmeda de junquillo, $\mathrm{C}=$ Polygono-Ludwigietum, $\mathrm{D}=$ totoral-ciperal, $\mathrm{E}=$ acuáticas, $\mathrm{VI}=$ valor de importancia; valores numéricos bajo los inventarios $=$ porcentajes de cobertura,$+=$ cobertura $<5 \%$ ).

Floristic structure of the herbaceous vegetation of National Reserve Lago Peñuelas $(\mathrm{A}=$ drought prairie, $\mathrm{B}=$ wet prairie of juncus, $\mathrm{C}=$ Polygono-Ludwigietum, $\mathrm{D}=$ totoral-cyperal, $\mathrm{E}=$ aquatics, $\mathrm{VI}=$ value of importance; numerical values under the inventories $=$ percentages of coverage, $+=$ coverage $<5 \%$ ).

\begin{tabular}{|c|c|c|c|c|c|c|c|c|c|c|c|}
\hline \multirow[b]{2}{*}{ Especies/Inventarios } & \multicolumn{3}{|c|}{$\mathrm{A}$} & \multicolumn{2}{|c|}{ B } & \multicolumn{2}{|c|}{$\mathrm{C}$} & \multicolumn{2}{|c|}{ D } & \multicolumn{2}{|l|}{$\mathrm{E}$} \\
\hline & 1 & 2 & 3 & 4 & 5 & 6 & 7 & 8 & 9 & 10 & VI \\
\hline Phyla canescens & 50 & 30 & - & 30 & 10 & 10 & 10 & - & - & - & 21,8 \\
\hline Hypochaeris radicata & 20 & 10 & 5 & + & 5 & - & - & - & - & - & 9,6 \\
\hline Bromus hordeaceus & 10 & 30 & 5 & - & - & - & - & - & - & - & 8,0 \\
\hline Gamochaeta spicata & 10 & 5 & - & + & + & - & - & - & - & - & 5,9 \\
\hline Baccharis linearis & 10 & - & 5 & - & + & - & - & - & - & - & 4,8 \\
\hline Agrostis capillaris & - & 10 & 10 & 30 & 40 & 20 & 10 & - & + & - & 20,6 \\
\hline Leontodon sax & - & 10 & 10 & - & - & - & - & - & - & - & 4,2 \\
\hline Avena barbata & - & + & + & - & - & - & - & - & - & - & 2,2 \\
\hline Cerastium fontan & - & + & - & - & - & - & - & - & - & - & 1,1 \\
\hline Rumex pulcher & - & + & 5 & 5 & - & + & - & - & - & - & 5,3 \\
\hline Baccharis salicifolia & - & - & + & - & - & + & - & - & + & - & 3,3 \\
\hline Rumex acetos & - & - & 5 & - & - & - & - & - & - & - & 1,6 \\
\hline Madia sativa & - & - & + & - & - & - & - & - & - & - & 1,1 \\
\hline Helenium aromatich & - & - & + & - & + & - & - & - & - & - & 2,2 \\
\hline Lactuca ser & - & - & + & + & - & - & - & - & - & - & 2,2 \\
\hline Briza minor & - & - & - & + & - & - & - & - & - & - & 1,1 \\
\hline Bartsia trixago & - & - & - & + & + & - & - & + & - & - & 3,3 \\
\hline Mentha pulegium & - & - & - & 5 & + & - & - & 10 & 5 & - & 6,3 \\
\hline Juncu: & - & - & - & 5 & - & + & - & + & - & - & 3,7 \\
\hline Juncl & - & - & - & + & 10 & + & - & - & - & + & 5,4 \\
\hline Cirsium vulgare & - & - & - & 5 & - & - & - & - & - & - & 1,6 \\
\hline Juncus pallescens & - & - & - & - & 25 & - & - & 5 & + & - & 6,5 \\
\hline Lythrum hyssopifolia & - & - & - & - & + & - & - & - & - & - & 1,1 \\
\hline Ludwigia peploides & - & - & - & - & 5 & 40 & 20 & 30 & 10 & - & 16,8 \\
\hline Paspalum dilatatum & - & - & - & - & - & 10 & + & - & - & - & 3,2 \\
\hline Distic & - & - & - & - & - & 20 & 20 & - & - & - & 6,5 \\
\hline Polygonum hydropip & - & - & - & - & - & + & - & 30 & 10 & + & 8,7 \\
\hline Cotula coronopifolia & - & - & - & - & - & + & 40 & - & - & - & 6,6 \\
\hline Melica violacea & - & - & - & - & - & + & - & - & - & - & 1,2 \\
\hline Cyperus eragrostis & - & - & - & - & - & - & + & 5 & + & - & 3,7 \\
\hline Echinochloa colona & - & - & - & - & - & - & + & - & - & - & 1,1 \\
\hline Verbena bonariensis & - & - & - & - & - & - & + & - & - & - & 1,1 \\
\hline Schoenoplectus californicus & - & - & - & - & - & - & - & 10 & 70 & - & 11,0 \\
\hline Carex & - & - & - & - & - & - & - & 5 & 5 & + & 4,2 \\
\hline Azolla filiculoides & - & - & - & - & - & - & - & 5 & - & 10 & 3,7 \\
\hline Hydrocotyle ranunculoides & - & - & - & - & - & - & - & + & - & + & 2,2 \\
\hline Eleocharis exigua & - & - & - & - & - & - & - & + & - & + & 2,2 \\
\hline Typha angustifolia & - & - & - & - & - & - & - & + & - & - & 1,1 \\
\hline Gunnera tinctoria & - & - & - & - & - & - & - & - & - & 10 & 2,1 \\
\hline Hydrocotyle modesta & - & - & - & - & - & - & - & - & - & + & 1,1 \\
\hline Blechnum chilense & - & - & - & - & - & - & - & - & - & + & 1,1 \\
\hline Total especies & 5 & 9 & 12 & 12 & 12 & 12 & 9 & 13 & 9 & 9 & - \\
\hline
\end{tabular}

En ambas comunidades (totoral y ciperal), por las condiciones de anegamiento del lugar, existe un predominio absoluto de las formas de vida hidrofíticas, tales como helófitos (plantas de pantano) e hidrófitos típicos.

h) Comunidades de acuáticas. Aunque estas comunidades estuvieron representadas sólo en un inventario (inventario 10, cuadro 4), correspondieron principalmente a especies hidrófitas, entre las que destacaron Azolla filiculoides, que es un pteridófito flotante libre, que constituye la asociación descrita como Lemo-Azolletum filiculoidis (Roussine y Negre 1952); además, la alta frecuencia de Hydrocotyle ranunculoides, $H$. modesta y Eleocharis exigua permiten deducir la existencia del Hydrocotyletum, pero la aplicación de un solo inventario es insuficiente para confirmar su presencia. Por ejemplo, en la parte más despejada de la vertiente del sector El Abrevadero, se forma un pequeño cuerpo de agua que está colonizado por especies acuáticas y palustres como: Azolla filiculoides, Gunnera tinctoria, Juncus cyperoides, Carex excelsa, Eleocharis exigua, Polygonum hydropiperoides, Hydrocotyle modesta e $H$. ranunculoides.

En resumen, se determinó un total de 10 asociaciones vegetales, de las cuales ocho son nuevas para el lugar. Estas últimas son las siguientes: a) bosque esclerófilo Peumo-Cryptocaryetum albae, b) bosque higrófilo Blepharocalyo-Myrceugenietum exsuccae, c) pradera seca Bromo-Lolietum, d) praderas húmedas Juncetum acutii y Polygono-Ludwigietum peploidis y e) comunidades de acuáticas Scirpetum californiae, Loto-Cyperetum eragrostidae y Lemno-Azolletum filiculoidis. Por otra parte, las especies que destacaron con altos valores de importancia en la vegetación leñosa (cuadro 3), es decir, mostraron alta frecuencia y cobertura en el área, fueron: Acacia caven, Agrostis capillaris, Schinus latifolia, Peumus boldus, Quillaja saponaria y Maytenus boaria. Las que destacaron en la vegetación herbácea (cuadro 4) fueron: Phyla canescens, Agrostis capillaris, Ludwigia peploides, Schoenoplectus californicus e Hypochaeris radicata.

\section{DISCUSIÓN}

Flora. Al comparar la riqueza florística general del lugar de estudio (337 especies) con otros realizados en áreas silvestres protegidas de Chile central, como las ubicadas en la Región del Maule: RN Los Bellotos del Melado con 297 especies (Arroyo et al. 2000), RN Los Ruiles con 139 especies, RN Los Queules con 104 especies (Arroyo et al. 2005); Región Metropolitana: Monumento Natural El Morado (300 especies) (Teillier 2003), RN Río Clarillo con aproximadamente 600 especies (Teillier et al. 2005); Región de Valparaíso: PN La Campana, 207 especies (Villaseñor 1980, 1986, Villaseñor y Serey 1980, 1981), están muy por debajo de lo registrado en la RN Lago Peñuelas, lo que demuestra la riqueza florística de 
esta área protegida. Una excepción es lo reportado por Teillier et al. (2005) para la RN Río Clarillo en la que prácticamente se duplica el número de especies, lo que se podría atribuir a una mayor variedad de ambientes y a su mayor superficie (13.085 ha).

El espectro biológico muestra el predominio de los terófitos, hemicriptófitos y criptófitos sobre el resto de las formas de vida, lo que está en concordancia con las condiciones de sequía del lugar, especialmente en la época estival, ya que los terófitos (plantas herbáceas de ciclos de vida corto, anuales o bienales) y los criptófitos (geófitos o plantas con órganos subterráneos perdurantes) representan muy bien este tipo de clima y son buenos indicadores ambientales. Por su parte, la abundancia de hemicriptófitos indica más bien intervención humana, ya que esta forma de vida acompaña al hombre y corresponde a plantas que son capaces de soportar el pisoteo y ramoneo de los animales domésticos (Cabrera y Willink 1973, Ramírez 1988, Grigera et al. 1996).

El origen fitogeográfico general de las plantas de la RN Lago Peñuelas indica que el $28 \%$ corresponde a especies introducidas, valor relativamente alto si se compara con la mayoría de los estudios mencionados anteriormente, donde los valores de especies alóctonas no superan el 20\%. De acuerdo a Hauenstein et al. (1988), la distribución porcentual en que las plantas alóctonas alcanzan valores entre 20 y $30 \%$, indica un grado relativamente alto de intervención antrópica. Ello, de acuerdo con González (2000), corresponde a la categoría de "medianamente intervenido". Esta mayor intervención se explica por la alta afluencia de visitantes y la presencia de animales domésticos en ciertos periodos del año, que permiten la llegada de terófitos y hemicriptófitos que en su mayoría corresponden a plantas de rápido crecimiento y fuertemente invasoras. A lo anterior se suma la diferente estructura del suelo bajo plantaciones de especies exóticas (Pinus radiata, Eucalyptus globulus) y la agresiva colonización de las acacias australianas (Acacia dealbata, A. melanoxylon) (ICSA 1980, CONAF 1994).

En todas estas áreas silvestres protegidas de la zona central chilena es destacable el alto porcentaje de especies endémicas, con valores superiores a $40 \%$, los que se incrementan al $70 \%$ al considerar sólo las plantas nativas; las introducidas en cambio, no superan el 30\% (Arroyo et al. 1995). Esta característica, entre otros elementos, destaca el valor de estas áreas como instancias de reserva y protección de la flora chilena y unidades de vegetación (Luebert y Becerra 1998).

Es importante destacar también el alto número de monocotiledóneas del sector, especialmente la abundancia de geófitas bulbosas, entre las cuales se encuentra la mayor parte de las especies con problemas de conservación. Vale recordar que éstas destacan por el colorido y hermosura de sus flores, siendo especialmente importantes en el sector Bajo Los Lirios (CONAF 1994), donde destacan las orquidáceas de gran colorido y variedad, las iridáceas, con lirios de los géneros Sisyrinchium y Olsynium y una especie "en peligro", como el azulillo (Calydorea xiphioides), muy escasa en toda la zona central. Destacan también otras especies más frecuentes pero no menos bellas, como el lahue (Herbertia lahue), y especies de los géneros Alstroemeria, Hippeastrum, Rhodophiala y Calceolaria de importancia estética para la recreación y uso en jardinería (Riedemann y Aldunate 2001, Muñoz y Moreira 2003). Este último sector requiere de una mayor atención de parte de la administración de la RN Lago Peñuelas, ya que al existir pocos senderos claramente definidos y señalizados, los visitantes recorren el lugar sin restricción, causando daños especialmente a este tipo de flora, debido al pisoteo y extracción de flores.

Vegetación. La fitosociología total del lugar señala la presencia de 20 asociaciones vegetales, de las cuales 10 son herbáceas, siete de matorral y tres boscosas; de ellas, 12 ya habían sido descritas en trabajos anteriores (ICSA 1980, CONAF 1986, 1994) y corresponden a las siguientes: pradera con Bromus mollis-Plantago lanceolata, pradera con Acacia caven, estepa con Stipa laevisima-Eryngium paniculatum-Satureja gilliesii, estepa de Piptochaetium montevidense - Stipa laevisima-Eyngium paniculatum, matorral con Baccharis linearis-Maytenus boaria, matorral abierto con Acacia caven-Maytenus boaria, matorral con Retanilla trinervia-Satureja gilliesii-Podanthus mitiqui, matorral alto con Escallonia pulverulenta-Cryptocarya alba, matorral con Retanilla trinervia-Colliguaja odorifera, matorral alto con Retanilla trinervia-Cryptocarya alba, matorral ripario con Otholobium glandulosum-Rubus ulmifolius y bosque abierto con Acacia caven-Maytenus boaria-Quillaja saponaria.

En el presente estudio se determinó un total de 10 comunidades vegetales, de las cuales ocho son nuevas para el lugar. Las dos que coinciden con las descritas en los trabajos anteriores corresponden al matorral abierto con Acacia caven-Maytenus boaria y matorral alto con Escallonia pulverulenta-Cryptocarya alba.

En relación al matorral abierto de espino, también conocido como la "estepa de espino o de acacia" (Pisano 1956, CONAF 1994), en sentido estricto no lo es, correspondiendo más bien a una sabana, tal como lo menciona Grau (1992), ya que la estepa comprende vegetación aislada con suelo desnudo a su alrededor y representa ambientes fríos. La sabana en cambio posee árboles o arbustos espinosos aislados y un rico estrato herbáceo (Cabrera y Willink 1973). Este estrato herbáceo rico en especies forrajeras permite, en ciertas épocas del año, el uso de este tipo de comunidad para el talaje en la RN Lago Peñuelas, actividad que requiere de una urgente revisión por parte de CONAF, ya que no se conocen sus efectos sobre la biodiversidad del lugar.

La dominancia de terófitos y geófitos en el espectro biológico de esta formación vegetal concuerda con las condiciones climáticas y niveles de precipitación del área en estudio, ya que estas formas de vida presentan adap- 
taciones morfológicas y fisiológicas a estas condiciones ambientales con periodos extensos de sequía, siendo, además, elementos importantes como alimento para la fauna silvestre. El menor porcentaje de fanerófitos se explica por los bajos niveles de precipitaciones del área y probablemente también a la tala realizada de la vegetación nativa leñosa en el pasado. La escasez de caméfitos se debe a que esta forma de vida está adaptada a condiciones de bajas temperaturas y de mayor altitud, condiciones que no reúne la RN Lago Peñuelas (Cabrera y Willink 1973, Ramírez 1988, Grigera et al. 1996).

En el matorral esclerófilo mixto son importantes los fanerófitos de características esclerófilas y los terófitos, lo que también señala la concordancia de este tipo de formación vegetal con las condiciones climáticas del área. La presencia importante de hemicriptófitos indica alteración antrópica (Hauenstein et al. 1988). Numerosos autores han realizado estudios ecofisiológicos en especies del matorral mediterráneo, que explican su adaptabilidad a este tipo de ambiente, entre ellos Mooney y Kummerow (1971) sobre la respuesta a la sequía de Flourensia thurifera, Kageneckia oblonga, Lithraea caustica y Proustia cinerea; Montenegro et al. (1979) sobre la dinámica de crecimiento de Colliguaja odorifera, Lithraea caustica, Satureja gilliesii y Retanilla trinervia; Araya y Ávila (1981) sobre el rebrote de especies del matorral afectado por el fuego; y Ávila et al. (1981) sobre el comportamiento de las especies del estrato herbáceo del matorral después de un incendio.

Respecto del bosque esclerófilo, dominado por fanerófitos y con presencia importante de terófitos y hemicriptófitos, también está en concordancia con el clima mediterráneo del área, ya que las especies presentan adaptaciones que les permiten sobrevivir un intenso estrés hídrico como, por ejemplo, la presencia de hojas esclerófilas (Mooney y Kummerow 1971), y los incendios que son frecuentes en el área. Para esto último, muchas desarrollan una gruesa peridermis y lignotúber, estructuras que les permiten rebrotar después de un siniestro (Araya y Ávila 1981). Esta comunidad fue clasificada por Oberdorfer (1960) en la clase Lithraeo causticae-Cryptocaryetea albae y se extiende desde los $31^{\circ} \mathrm{S}$ con el límite del territorio templado (Aguilella y Amigo 2001). El área con este tipo de vegetación es la que sin duda ha sido más afectada por la acción humana (Balduzzi et al. 1981, 1982).

Por otra parte, la presencia de remanentes de bosque higrófilo, que requiere condiciones de mayor humedad y pluviometría, presenta un predominio absoluto de fanerófitos y nanofanerófitos (arbustos) sobre las otras formas de vida, lo cual estaría indicando que el área presentaría altos niveles de pluviometría, lo que no es el caso. La explicación a la presencia de este bosque de características higrófilas en el lugar radica en que se desarrolla sobre una vertiente ubicada en el sector Vega del Álamo, también llamado el "abrevadero de caballos", que genera abundante agua durante todo el año, la que es capaz de mantener dicha comunidad de características azonales (Ramírez et al. 1996).

Las praderas secas se caracterizan por el predominio de hemicriptófitos que indican una fuerte acción antrópica, ya que dichas formas de vida están adaptadas para soportar el pisoteo y ramoneo de los animales introducidos por el hombre, y de terófitos, que indican condiciones de sequía (Hauenstein et al. 1988, Ramírez 1988). En las praderas húmedas, en cambio, dominan los criptófitos y hemicriptófitos.

Respecto del totoral, es la asociación palustre más abundante y variable del centro-sur de Chile, colonizando bañados y riberas de cuerpos acuáticos lóticos y lénticos de poca profundidad (Ramírez y Añazco 1982, Ramírez et al. 1987, San Martín et al. 1993). Igual situación ocurre con el ciperal, que habitualmente acompaña al totoral, formando una franja característica más seca, cuyas especies principales Cyperus eragrostis y Carex excelsa poseen hojas de borde cortante, de donde deriva su nombre común de "cortaderas" (Hauenstein et al. 2002, 2005a).

La comunidad de hidrófitas corresponde al LemnoAzolletum que caracteriza a ambientes acuáticos eutroficados (Palma et al. 1978) y que Ramírez et al. (1987) mencionan como muy abundante en lagunas de la zona central de Chile; asimismo, especies como Hydrocotyle modesta e $H$. ranunculoides son indicadoras de contaminación orgánica por nitrógeno (Hauenstein et al. 2005b), lo que corroboraría altos niveles tróficos del lago Peñuelas ${ }^{1}$; por otra parte, este cuerpo de agua y su flora asociada es el hábitat de una rica avifauna (Strang 1983).

En trabajos anteriores realizados en la RN Lago Peñuelas (ICSA 1980, CONAF 1986, 1994) se describe la presencia de sólo tres unidades de vegetación que reconocen como "comunidades naturales": a) matorral ripícola, b) bosque esclerófilo mixto y c) bosque y matorral espinoso caducifolio; las que corresponden más bien a grandes unidades de vegetación. Al interior de ellas se insertan 12 "unidades florísticas" o comunidades basadas en el "sistema básico de clasificación de la vegetación nativa chilena" (Gajardo 1983, 1995), ya señaladas anteriormente.

Los resultados de este trabajo amplían los antecedentes florísticos y sinecológicos de la RN Lago Peñuelas, destacando su riqueza tanto florística como vegetacional, lo que refuerza la necesidad de mantener esta área protegida como parte de la Reserva de la Biósfera ya establecida, aunque es urgente una revisión de algunas medidas de manejo, tales como: a) elaboración de senderos interpretativos, b) mejoramiento de los senderos existentes, c) definición de áreas de uso y su ordenamiento (áreas de conservación, preservación, etc.), d) eliminar las autorizaciones de uso para talaje de animales, e) recuperar áreas naturales, eliminando o reduciendo las plantaciones forestales.

\footnotetext{
Irma Vila, Facultad de Ciencias, Universidad de Chile. Comunicación personal.
} 
A este respecto, Arroyo et al. (2005), entre otras medidas, plantean la urgente necesidad de completar los inventarios florísticos de todas las áreas protegidas de Chile central; asimismo, sugieren analizar estas áreas a escala de polígonos pequeños para evaluar su relevancia biológica y vulnerabilidad, elementos que deben ser la base para una estrategia de conservación integrada. De igual forma, es importante lo manifestado por Armesto et al. (2002) y Simonetti (2004) respecto de la necesidad de aumentar el número de áreas protegidas tanto públicas como privadas, incrementar la superficie de las ya existentes y la necesidad de emplear el ambiente intervenido que las rodea, con el objeto de crear zonas de interconectividad entre ellas que puedan servir como corredores biológicos. Estas unidades deberían considerar la representación de los distintos tipos de vegetación (Luebert y Becerra 1998). También es interesante considerar la propuesta de Elórtegui y MoreiraMuñoz (2002) para el Parque Nacional La Campana, en el sentido de zonificar áreas con distintos tipos de usos. Bajo este marco, Simonetti (1995) plantea la necesidad de contar con un modelo que planifique el ordenamiento de estas áreas protegidas, que vincule la necesidad de la conservación de recursos naturales y su aprovechamiento sustentable, lo cual sería una herramienta fundamental para apoyar y definir cuáles, dónde y cómo se realizarán las actividades posibles de implementar, para que éstas sean compatibles con la conservación de la biodiversidad.

\section{AGRADECIMIENTOS}

Los autores agradecen a CONAF Región de Valparaíso por el financiamiento de este estudio, y a las siguientes personas: J. Meza, S. Huaiquinao de CONAF y a E. I. Hauenstein (Jr.) por su colaboración en terreno.

\section{REFERENCIAS}

Aguilella A, J Amigo. 2001. Transectos de vegetación en el centro-sur de Chile. In Gómez-Mercado T, J Mota-Poveda eds. Vegetación y cambios climáticos. España. Universidad de Almería. p. 87-101.

Araya S, G Ávila. 1981. Rebrote de arbustos afectados por el fuego en el "Matorral chileno". Anales del Museo de Historia Natural de Valparaíso 14: 99-105.

Armesto J, C Papic, P Pliscoff. 2002. Relevancia de las pequeñas áreas silvestres para la conservación de la biodiversidad en el bosque nativo. Ambiente y Desarrollo 18: 44-50.

Arroyo MTK, L Cavieres, C Marticorena, M Muñoz. 1995. Convergence in the mediterranean floras of central Chile and California: Insights from comparative biogeography. In Arroyo MTK, M Fox, P Zedler eds. Ecology and biogeography of mediterranean ecosystems in Chile, California, and Australia. New York. Springer-Verlag. p. 43-88.

Arroyo MTK, O Matthei, C Marticorena, M Muñoz, F Pérez, AM Humaña. 2000. The vascular plant flora of the Bellotos del Melado National Reserve, VII Region, Chile: a documented checklist. Gayana Botánica 57: 117-139.
Arroyo MTK, O Matthei, M Muñoz-Schick, JJ Armesto, P Pliscoff, F Pérez, C Marticorena. 2005. Flora de cuatro Reservas Nacionales en la Cordillera de la Costa de la VII Región $\left(35^{\circ}-36^{\circ} \mathrm{S}\right)$, Chile, y su papel en la protección de la biodiversidad regional. In Smith-Ramírez C, JJ Armesto, C Valdovinos eds. Historia, biodiversidad y ecología de los bosques costeros de Chile. Santiago, Chile. Editorial Universitaria. p. 225-244.

Ávila G, ME Aljaro, B Silva. 1981. Observaciones en el estrato herbáceo del matorral después del fuego. Anales Museo de Historia Natural de Valparaíso 14: 107-113.

Baeza M, E Barrera, J Flores, C Ramírez, R Rodríguez. 1998. Categorías de conservación de Pteridophyta nativas de Chile. Boletín Museo Nacional de Historia Natural, Chile 47: 23-46.

Balduzzi A, I Serey, R Tomaselli, R Villaseñor. 1981. New phytosociological observations on the Mediterranean type of climax vegetation of central Chile. Atti Istituto Botanico Laboratori Crittogamico di Pavia, serie 6, 14: 93-112.

Balduzzi A, R Tomaselli, I Serey, R Villaseñor. 1982. Degradation of the mediterranean type of vegetation in central Chile. Ecología Mediterránea 8: 223-240.

Belmonte E, L Faúndez, J Flores, A Hoffmann, M Muñoz, S Teillier. 1998. Categorías de conservación de cactáceas nativas de Chile. Boletín Museo Nacional de Historia Natural, Chile 47: 69-89.

Benoit IL ed. 1989. Libro rojo de la flora terrestre de Chile. Santiago, Chile. CONAF. 157 p.

Braun-Blanquet J. 1964. Pflanzensoziologie-Grundzuge der Vegetationskunde. Viena, Austria. Springer Verlag. 865 p.

Braun-Blanquet J. 1979. Fitosociología. Base para el estudio de las comunidades vegetales. Madrid, España. Blume. 686 p.

Cabrera AL, A Willink. 1973. Biogeografía de América Latina. Washington D.C. Serie de Biología, Monografía No 13 , Programa Regional de Desarrollo Científico y Tecnológico, Departamento de Asuntos Científicos, Secretaría General de la Organización de los Estados Americanos. 120 p.

Cavieres LA, M Mihoc, A Marticorena, C Marticorena, O Matthei, FA Squeo. 2001. Determinación de áreas prioritarias para la conservación: análisis de parsimonia de endemismos (PAE) en la flora de la IV Región de Coquimbo. In Squeo FA, G Arancio, JR Gutiérrez eds. Libro rojo de la flora nativa y de los sitios prioritarios para su conservación: Región de Coquimbo. La Serena, Chile. Universidad de La Serena. p. $159-170$.

CONAF (Corporación Nacional Forestal, CL). 1986. Plan de manejo, Reserva Forestal Lago Peñuelas. Santiago, Chile. Corporación Nacional Forestal. 20 p. (Documento de Trabajo $\mathrm{N}^{\circ} 77$ )

CONAF (Corporación Nacional Forestal, CL). 1992. Parques Nacionales y Monumentos Naturales de Chile. Santiago, Chile. Corporación Nacional Forestal. 2 p.

CONAF (Corporación Nacional Forestal, CL). 1994. Antecedentes de la riqueza florística del sector Bajo los Lirios. Reserva Nacional Lago Peñuelas. Santiago, Chile. Corporación Nacional Forestal 15 p.

CONAF-CONAMA-BIRF (Corporación Nacional Forestal, CL-Comisión Nacional del Medio Ambiente, CL-Banco Internacional de Reconstrucción y Fomento, CL). 1999. Catastro y evaluación de recursos vegetacionales nativos de Chile. Informe Regional Quinta Región. Santiago, Chile. Proyecto CONAF-CONAMA-BIRF. 141 p. 
DI Castri F, E Hajek. 1976. Bioclimatología de Chile. Santiago, Chile. Universidad Católica de Chile. 128 p.

Ellenberg H, D Mueller-Dombois. 1966. A key to Raunkiaer plant life forms with revised subdivisions. Ber. Geob. Inst. ETH Stiftung Rubel, Zurich 37: 56-73.

Elórtegui S, A Moreira-Muñoz eds. 2002. Parque Nacional La Campana: Origen de una Reserva de la Biósfera en Chile Central. Santiago, Chile. Taller La Era. 176 p.

Gajardo R. 1983. Sistema básico de clasificación de la vegetación nativa chilena. Santiago, Chile. Corporación Nacional Forestal/Universidad de Chile. 314 p.

Gajardo R. 1995. La vegetación natural de Chile. Clasificación y distribución. Santiago, Chile. Editorial Universitaria. $165 \mathrm{p}$.

González A. 2000. Evaluación del recurso vegetacional en la cuenca del río Budi, situación actual y propuestas de manejo. Tesis Licenciatura en Recursos Naturales. Temuco, Chile. Facultad de Ciencias, Universidad Católica de Temuco. 110 p.

Grau J. 1992. La zona central de Chile. In Grau J, G Zizka eds. Flora silvestre de Chile. Palmengarten, Sonderheft 19: 1-154.

Grigera D, C Brion, JO Chiapella, MS Pillado. 1996. Las formas de vida de las plantas como indicadores de factores ambientales. Medio Ambiente 13: 11-29.

Hauenstein E, E Parada. 1975. Curso Práctico de Botánica General. Temuco, Chile. Edic. Universitarias de La Frontera. $110 \mathrm{p}$.

Hauenstein E, C Ramírez, M Latsague, D Contreras. 1988. Origen fitogeográfico y espectro biológico como medida del grado de intervención antrópica en comunidades vegetales. Medio Ambiente 9: 140-142.

Hauenstein E, M González, F Peña, A Muñoz. 2002. Clasificación y caracterización de la flora y vegetación de los humedales de la costa de Toltén (IX Región, Chile). Gayana Botánica 59: 87-100.

Hauenstein E, M González, F Peña-Cortés, A Muñoz-Pedreros. 2005a. Diversidad vegetal en humedales costeros de la Región de La Araucanía. In Smith-Ramírez C, JJ Armesto, C Valdovinos eds. Historia, biodiversidad y ecología de los bosques costeros de Chile. Santiago, Chile. Editorial Universitaria. p. 197-205.

Hauenstein E, M González, F Peña-Cortés, L Falcón. 2005b. Plantas indicadoras de eutrofización en lagos del sur de Chile. In Vila I, J Pizarro eds. Eutrofización de lagos y embalses. Programa Iberoamericano de Ciencia y Tecnología para el Desarrollo (CYTED-Universidad de Chile). Santiago, Chile. Patagonia Impresores. p. 119-133.

Hoffmann A. 1978. Flora silvestre de Chile, zona central. Santiago, Chile. Fundación Claudio Gay. 255 p.

Hoffmann A. 1991. Flora silvestre de Chile, zona araucana. Santiago, Chile. $2^{\mathrm{a}}$ ed. Fundación Claudio Gay. 257 p.

Hoffmann A, MK Arroyo, F Liberona, M Muñoz, J Watson. 1998. Plantas altoandinas en la flora silvestre de Chile. Santiago, Chile. Fundación Claudio Gay. 281 p.

ICSA (Ingenieros Consultores Asociados S.A.). 1980. Estudio de proposiciones de potencialidades de desarrollo integral de la Reserva Forestal Lago Peñuelas. Santiago, Chile. ICSA-CONAF. 187 p.

IPNI (International Plant Names Index). 2008. The International Plant Names Index. Consultado 10 jul. 2008. Disponible en http://www.ipni.org/
Köppen W. 1931. Die klimate der Erde. Grundiss der Klimakunde. Berlin, Alemania. Gruyter. 182 p.

Looser G. 1944. Anotaciones fitosociológicas sobre la región de Quintero. Revista Universitaria 29: 27-33.

Luebert F, P Becerra. 1998. Representatividad vegetacional del Sistema Nacional de Áreas Silvestres Protegidas del Estado (SNASPE) en Chile. Ambiente y Desarrollo 14(2): 62-69.

Luebert F, P Pliscoff. 2006. Sinopsis bioclimática y vegetacional de Chile. Santiago, Chile. Editorial Universitaria. 307 p.

Marticorena C, M Quezada. 1985. Catálogo de la flora vascular de Chile. Gayana Botanica 42: 1-155.

Marticorena C, R Rodríguez eds. 1995. Flora de Chile. Vol. 1. Pteridophyta- Gymnospermae. Concepción, Chile. Universidad de Concepción. 351 p.

Marticorena C, R Rodríguez eds. 2001. Flora de Chile. Vol. 2(1). Winteraceae-Ranunculaceae. Concepción, Chile. Universidad de Concepción. 99 p.

Marticorena C, R Rodríguez eds. 2003. Flora de Chile. Vol. 2 (2). Berberidaceae-Betulaceae. Concepción, Chile. Universidad de Concepción. 93 p.

Marticorena C, R Rodríguez eds. 2005. Flora de Chile.Vol. 2 (3). Plumbaginaceae-Malvaceae. Concepción, Chile. Universidad de Concepción. 128 p.

Marticorena C, C Von Bohlen, M Muñoz, MTK Arroyo. 1995. Dicotiledóneas. In Simonetti JA, MTK Arroyo, AE Spotorno, E Lozada eds. Diversidad biológica de Chile. Santiago, Chile. Artegrama. p. 77-89.

Matthei O. 1995. Manual de las malezas que crecen en Chile. Santiago, Chile. Alfabeta Impresores. 545 p.

Mittermeier RA, N Myers, JB Thomsen, GAB Da Fonseca, S Olivieri. 1998. Biodiversity hotspots and major tropical wilderness area: approaches to setting conservation priorities. Conservation Biology 12: 516-520.

Montenegro G, ME Aljaro, J Kummerow. 1979. Growth dynamics of chilean matorral shrubs. Botanical Gazette 140: 114-119.

Mooney HA, J Kummerow. 1971. The comparative water economy of representative evergreen sclerophyll and drought deciduous shrubs of Chile. Botanical Gazette 132: 246-252.

Mueller-Dombois D, E Ellenberg. 1974. Aims and methods of vegetation ecology. New York. John Wiley \& Sons. 547 p.

Muñoz C. 1966. Sinopsis de la flora chilena. 2a ed. Santiago, Chile. Universidad de Chile. 500 p.

Muñoz M, A Moreira. 2003. Alstroemerias de Chile: diversidad, distribución y conservación. Santiago, Chile. Taller La Era. 139 p.

Myers N, RA Mittermeier, CG Mittermeier, GA Da Fonseca, J Kent. 2000. Biodiversity hotspots for conservation priorities. Nature 403: 853-858.

Navas ME. 1973. Flora de la cuenca de Santiago de Chile. Tomo I. Santiago, Chile. Universidad de Chile. 299 p.

Navas ME. 1976. Flora de la cuenca de Santiago de Chile. Tomo II. Santiago, Chile. Universidad de Chile. 559 p.

Navas ME. 1979. Flora de la cuenca de Santiago de Chile. Tomo III. Santiago, Chile. Universidad de Chile. 509 p.

Novoa P, J Espejo, M Cisternas, M Rubio, E Domínguez. 2006. Guía de campo de las orquídeas chilenas. Concepción, Chile. Corporación Chilena de la Madera. 120 p.

Oberdorfer E. 1960. Planzensoziologishe Studien in Chile-Ein Vergleich mit Europa. Weinheim. Cramer. 208 p. 
Oltremari J. 2002. Las áreas protegidas y la conservación de la diversidad biológica. Santiago, Chile. Universidad Católica de Chile. 11 p.

Palma B, C San Martín, M Rosales, L Zúñiga, C Ramírez. 1978. Distribución espacial de la flora y vegetación acuática y palustre del estero Marga-Marga en Chile central. Anales del Instituto de Ciencias del Mar y Limnología Universidad Nacional Autónoma de México 14(2): 125-132.

Pisano E. 1956. Esquema de clasificación de las comunidades vegetales de Chile. Agronomía 2: 30-33.

Ramírez C. 1988. Formas de vida, fitoclimas y formaciones vegetales. El Árbol...Nuestro Amigo 4: 33-37.

Ramírez C, N Añazco. 1982. Variaciones estacionales en el desarrollo de Scirpus californicus, Typha angustifolia y Phragmites communis en pantanos valdivianos, Chile. Agro Sur 10: 111-123.

Ramírez C, R Westermeier. 1976. Estudio de la vegetación espontánea del Jardín Botánico de la Universidad Austral de Chile (Valdivia), como ejemplo de tabulación fitosociológica. Agro Sur 4: 93-105.

Ramírez C, C San Martín, J San Martín. 1996. Estructura florística de los bosques pantanosos de Chile central. In Armesto JJ, MTK Arroyo, C Villagrán eds. Ecología de los bosques nativos de Chile. Santiago, Chile. Editorial Universitaria. p. 215-234.

Ramírez C, J San Martín, C San Martín, D Contreras. 1987. Estudio florístico y vegetacional de la Laguna El Peral, Quinta Región de Chile. Revista Geográfica de Valparaíso 18: $105-120$.

Ravenna P, S Teillier, J Macaya, R Rodríguez, O Zöllner. 1998. Categorías de conservación de las plantas bulbosas nativas de Chile. Boletín Museo Nacional de Historia Natural, Chile 47: 47-68.

Riedemann P, G Aldunate. 2001. Flora nativa de valor ornamental, identificación y propagación. Chile, zona centro. Santiago, Chile. Andrés Bello. 566 p.

Roussine N, R Negre. 1952. Les groupements vegetaux de la France mediterraneenne. Montpellier. CNRS. 297 p.

San Martín C, R Medina, P Ojeda, C Ramírez. 1993. La biodiversidad vegetacional del Santuario de la Naturaleza Río Cruces (Valdivia, Chile). Acta Botánica Malacitana 18: 259-279.

San Martín C, C Ramírez, H Rubilar. 2002. Ecofisiología de los pantanos de cortadera en Valdivia, Chile. Ciencia $e$ Investigación Agraria 29: 171-179.
Simonetti JA. 1995. Diversidad biológica: algo más que nombres, algo más que números. In Simonetti JA, MTK Arroyo, AE Spotorno, E Lozada eds. Diversidad biológica de Chile. Santiago, Chile. Artegrama. p. 1-4.

Simonetti JA. 2004. Conectar para conservar. Ambiente y Desarrollo 20: 2-4.

Strang C. 1983. Las aves del lago Peñuelas. Chile Forestal 95: 20-22.

Steubing L, C Ramírez, M Alberdi. 1980. Energy content of water and bog plant associations in the region of Valdivia (Chile). Vegetatio 43: 153-161.

Steubing L, R Godoy, M Alberdi. 2002. Métodos de Ecología vegetal. Valdivia, Chile. Colección textos Universitarios, Universidad Austral de Chile. 345 p.

Teillier S. 2003. Flora del Monumento Natural El Morado: Addenda et Corrigenda. Gayana Bot. 60: 94-101.

Teillier S, G Aldunate, P Riedemann, H Niemeyer. 2005. Flora de la Reserva Nacional Río Clarillo. Guía de identificación de especies. Santiago, Chile. Impresos Socías. 367 p.

UICN (Unión Internacional para la Conservación de la Naturaleza, CH). 1994. Directrices para las categorías de manejo de áreas protegidas. Gland, Suiza. Unión Mundial para la Conservación de la Naturaleza (UICN). 261 p.

Villaseñor R. 1980. Unidades fisionómicas y florísticas del Parque Nacional La Campana. Anales del Museo de Historia Natural de Valparaíso 13: 65-70.

Villaseñor R. 1986. Guía para el reconocimiento de las especies arbóreas y arbustivas más frecuentes en el Parque Nacional La Campana. Valparaíso, Chile. CONAF-Universidad de Playa Ancha. 190 p.

Villaseñor R, I Serey. 1980-1981. Estudio fitosociológico de la vegetación del Cerro La Campana (Parque Nacional La Campana) en Chile central. Atti Ist. Bot. Lab. Critt. Univ. Pavia 6: 69-91.

Weber C. 1986. Conservación y uso racional de la naturaleza, en áreas protegidas. Ambiente y Desarrollo 2: 165-181.

Wikum D, GF Shanholtzer. 1978. Application of the BraunBlanquet cover-abundance scale for vegetation analysis in land development studies. Environmental Management 2: 323-329.

Zöllner O, M Olivares, ME Varas. 1995. El género Fumaria L. (Fumariaceae) en la zona central de Chile. Anales del Museo de Historia Natural de Valparaíso 23: 21-31. 
Anexo 1. Catálogo florístico de la Reserva Nacional Lago Peñuelas (FV = forma de vida, $\mathrm{OF}=$ origen geográfico, $\mathrm{EC}=$ estado de conservación, $\mathrm{REG}=$ registro de la especie, $\mathrm{Hi}=$ hidrófito, $\mathrm{Ge}=$ geófito, $\mathrm{F}=$ fanerófito, $\mathrm{Nf}=$ nanofanerófito, Hc $=$ hemicriptófito, $\mathrm{C}$ = caméfito, $\mathrm{N}$ = nativo, $\mathrm{I}=$ introducido, $\mathrm{P}=$ en peligro, $\mathrm{V}=$ vulnerable, $\mathrm{IC}=$ insuficientemente conocida, $1=$ especie registrada en este estudio, 2 = en ICSA (1980), 3 = en CONAF (1986), 4 = en CONAF (1994), 5 = en Zöllner et al. $(1995)$, $6=$ en Novoa et al. (2006)).

Checklist of the flora of National Reserve Lago Peñuelas $(\mathrm{FV}=$ life form, OF = geographic origin, EC = state of conservation, $\mathrm{REG}=$ species record $\mathrm{Hi}=$ hydrophytes, $\mathrm{Ge}=$ geophytes, $\mathrm{F}=$ phanerophytes, $\mathrm{Nf}=$ nanophanerophytes, $\mathrm{Hc}=$ hemicryptophytes, $\mathrm{C}=$ chamaephytes, $\mathrm{N}=$ native, $\mathrm{I}=$ introduced, $\mathrm{P}=$ threatened, $\mathrm{V}=$ vulnerable, $\mathrm{IC}=$ data deficient, $1=$ species recorded in this study, $2=$ in ICSA $(1980), 3=$ in CONAF (1986), 4 = in CONAF (1994), 5 = in Zöllner et al. (1995), $6=$ in Novoa et al. (2006)).

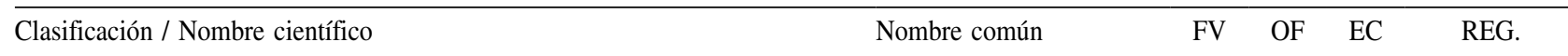

\section{PTERIDOPHYTA}

- Adiantaceae

Adiantum chilense Kaulf. var. hirsutum Hook. et Grev.

Adiantum excisum Kunze

Adiantum scabrum Kaulf.

Cheilanthes hypoleuca (Kunze) Mett.

- Azollaceae

Azolla filiculoides Lam.

- Blechnaceae

Blechnum chilense (Kaulf.) Mett.

Blechnum hastatum Kaulf.

- Equisetaceae

Equisetum bogotense Kunth

\begin{tabular}{|c|c|c|c|c|}
\hline Palito negro & $\mathrm{Ge}$ & $\mathrm{N}$ & & $1,2,4$ \\
\hline Palito negro & $\mathrm{Ge}$ & $\mathrm{N}$ & & 2 \\
\hline Palito negro & $\mathrm{Ge}$ & $\mathrm{N}$ & & 2,4 \\
\hline Doradilla & $\mathrm{Ge}$ & $\mathrm{N}$ & & 4 \\
\hline Flor del pato & $\mathrm{Hi}$ & $\mathrm{N}$ & & 1,2 \\
\hline Costilla de vaca & $\mathrm{Ge}$ & $\mathrm{N}$ & IC & 1 \\
\hline Palmilla & $\mathrm{Ge}$ & $\mathrm{N}$ & & $1,2,3,4$ \\
\hline Hierba del platero & $\mathrm{Ge}$ & $\mathrm{N}$ & & 3 \\
\hline
\end{tabular}

GYMNOSPERMAE (PINOPHYTA)

- Cupressaceae

Cupressus macrocarpa Hartw.

- Pinaceae

Pinus radiata D. Don

Ciprés de Monterrey

F I

1,3

Pino insigne

F I

$1,3,4$

ANGIOSPERMAE (MAGNOLIOPHYTA)

A) Dicotyledoneae (Magnoliopsida)

- Acanthaceae

Stenandrium dulce (Cav.) Nees

- Anacardiaceae

Lithraea caustica (Molina) Hook. et Arn.

Schinus latifolia (Gillies ex Lindl.) Engler

Schinus polygama (Cav.) Cabrera

- Apiaceae

Asteriscium chilense Cham. et Schltdl.

Bowlesia uncinata Colla

Cyclospermum laciniatum (DC.) Constance

Conium maculatum L.

Eryngium paniculatum Cav. et Dombey ex Delaroche

Hydrocotyle modesta Cham. et Schlecht.

Hydrocotyle ranunculoides L.f.

Sanicula crassicaulis Poepp. ex DC.

Torilis nodosa (L.) Gaertn.

- Asteraceae

Acrisione denticulata (Hook. et Arn.) B.Nord.

Ageratina glechonophylla (Less.) R.M.King et H.Rob.

Anthemis cotula L.

Aristeguietia salvia (Colla) R.M.King et H.Rob.

$\begin{array}{lccc}\text { Hierba de la piñada } & \mathrm{Hc} & \mathrm{N} & 4 \\ \text { Litre } & \mathrm{Nf} & \mathrm{N} & 1,2,3,4 \\ \text { Molle } & \mathrm{F} & \mathrm{N} & 1,2,3,4 \\ \text { Huingán, borocoi } & \mathrm{F} & \mathrm{N} & 1,3 \\ & & & \\ \text { Anicillo } & \mathrm{Hc} & \mathrm{N} & 2,4 \\ \text { Barba de gato } & \mathrm{Te} & \mathrm{N} & 2 \\ \text { Capuchilla } & \mathrm{Te} & \mathrm{N} & 1,2 \\ \text { Cicuta } & \mathrm{Te} & \mathrm{I} & 1,2,4 \\ \text { Cardoncillo } & \mathrm{Hc} & \mathrm{N} & 1,2,4 \\ \text { Sombrero de agua } & \mathrm{Hi} & \mathrm{N} & 1 \\ \text { Sombrero de agua } & \mathrm{Hi} & \mathrm{N} & 1,2 \\ \text { Pata de león } & \mathrm{Hc} & \mathrm{N} & 2 \\ \text { s.n. } & \mathrm{Te} & \mathrm{N} & 2 \\ & & & \\ \text { Palpalén } & \mathrm{F} & \mathrm{N} & 2,3 \\ \text { Barbón } & \mathrm{Nf} & \mathrm{N} & 2,4 \\ \text { Manzanillón } & \mathrm{Te} & \mathrm{I} & 1,2,4 \\ \text { Salvia macho } & \mathrm{Nf} & \mathrm{N} & 1,2 \\ & & & \end{array}$

Continúa 
Continuación de Anexo 1

\begin{tabular}{|c|c|c|c|c|c|}
\hline Clasificación / Nombre científico & Nombre común & $\mathrm{FV}$ & OF & $\mathrm{EC}$ & REG. \\
\hline Baccharis linearis (Ruiz et Pav.) Pers. & Romerillo & $\mathrm{Nf}$ & $\mathrm{N}$ & & $1,2,3,4$ \\
\hline Baccharis macraei Hook. et Arn. & Vautro & $\mathrm{Nf}$ & $\mathrm{N}$ & & $2,3,4$ \\
\hline Baccharis paniculata DC. & Chilca & $\mathrm{Nf}$ & $\mathrm{N}$ & & 2 \\
\hline Baccharis rhomboidalis Remy & Vautro & $\mathrm{Nf}$ & $\mathrm{N}$ & & 1 \\
\hline Baccharis salicifolia (Ruiz et Pav.) Pers. & Chilca & $\mathrm{Nf}$ & $\mathrm{N}$ & & 1,2 \\
\hline Bahia ambrosioides Lag. & Manzanilla cimarrona & $\mathrm{Nf}$ & $\mathrm{N}$ & & 2 \\
\hline Carduus pycnocephalus L. & Cardilla & $\mathrm{Te}$ & I & & 1 \\
\hline Centaurea melitensis L. & Cizaña, abrepuño & $\mathrm{Te}$ & I & & 1 \\
\hline Centaurea solstitialis L. & Cardo amarillo & $\mathrm{Te}$ & I & & 4 \\
\hline Chaetanthera linearis Poepp. ex Less. & s.n. & $\mathrm{Te}$ & $\mathrm{N}$ & & 2 \\
\hline Chaetanthera microphylla (Cass.) Hook. et Arn. & s.n. & $\mathrm{Te}$ & $\mathrm{N}$ & & 4 \\
\hline Cirsium vulgare (Savi) Ten. & Cardo negro & $\mathrm{Te}$ & I & & 1,2 \\
\hline Conyza sumatrensis (Retz.) E.Walker var. floribunda (Kunth) J.B.Marshall & s.n. & $\mathrm{Te}$ & $\mathrm{N}$ & & 1 \\
\hline Cotula australis (Sieber ex Spreng.) Hook.f. & s.n. & $\mathrm{Te}$ & I & & 2 \\
\hline Cotula coronopifolia $\mathrm{L}$. & Botón de oro & $\mathrm{Hc}$ & I & & $1,3,4$ \\
\hline Cynara cardunculus L. & Cardo penquero & $\mathrm{Hc}$ & I & & 1,2 \\
\hline Erigeron fasciculatus Colla & s.n. & $\mathrm{Hc}$ & $\mathrm{N}$ & & 2 \\
\hline Facelis retusa (Lam.) Sch. Bip. & s.n. & $\mathrm{Te}$ & $\mathrm{N}$ & & 2 \\
\hline Gamochaeta coarctata (Willd.) Kerguélen & Nafalium & $\mathrm{Te}$ & $\mathrm{N}$ & & 1 \\
\hline Haplopappus poeppigianus (Hook. et Arn.) A.Gray & s.n. & $\mathrm{C}$ & $\mathrm{N}$ & & 4 \\
\hline Haplopappus velutinus Remy subsp. illinitus (Phil.) Klingenb. & s.n. & $\mathrm{C}$ & $\mathrm{N}$ & & 2 \\
\hline Helenium aromaticum (Hook) L.H.Bailey & Manzanilla & $\mathrm{Te}$ & $\mathrm{N}$ & & 1 \\
\hline Hypochaeris glabra $\mathrm{L}$. & Hierba del chancho & $\mathrm{Te}$ & I & & 1,2 \\
\hline Hypochaeris radicata $\mathrm{L}$. & Hierba del chancho & $\mathrm{Hc}$ & I & & 1,4 \\
\hline Hypochaeris scorzonerae (DC.) F. Muell. & Escorzonera & $\mathrm{Hc}$ & $\mathrm{N}$ & & 4 \\
\hline Lactuca serriola $\mathrm{L}$. & Lechuguilla & $\mathrm{Te}$ & I & & 1 \\
\hline Leontodon saxatilis Lam. & Chinilla & $\mathrm{Hc}$ & I & & 1 \\
\hline Leucheria cerberoana Remy & s.n. & $\mathrm{Te}$ & $\mathrm{N}$ & & 4 \\
\hline Logfia gallica (L.) Coss. et Germ. & s.n. & $\mathrm{Te}$ & I & & 1 \\
\hline Madia sativa Molina & Melosa, pegajosa & $\mathrm{Te}$ & $\mathrm{N}$ & & 1,4 \\
\hline Matricaria chamomilla L. & Manzanilla & $\mathrm{Te}$ & I & & 4 \\
\hline Microseris pygmaea D. Don & s.n. & $\mathrm{Te}$ & $\mathrm{N}$ & & $1,2,4$ \\
\hline Mutisia latifolia D. Don & Clavel del campo & $\mathrm{F}$ & $\mathrm{N}$ & & 2 \\
\hline Mutisia subulata Ruiz et Pav. & Hierba del jote & $\mathrm{F}$ & $\mathrm{N}$ & & 2 \\
\hline Podanthus mitiqui Lindl. & Mitique & $\mathrm{Nf}$ & $\mathrm{N}$ & & $1,2,3$ \\
\hline Proustia pyrifolia DC. & Voqui blanco & $\mathrm{F}$ & $\mathrm{N}$ & & $1,2,3$ \\
\hline Gnaphalium cheiranthifolium Lam. & Viravira & $\mathrm{Hc}$ & $\mathrm{N}$ & & 2 \\
\hline Senecio adenotrichius DC. & s.n. & $\mathrm{Hc}$ & $\mathrm{N}$ & & 2 \\
\hline Silybum marianum (L.) Gaertn. & Cardo blanco & $\mathrm{Te}$ & I & & 1,2 \\
\hline Soliva sessilis Ruiz et Pav. & Dicha & $\mathrm{Te}$ & $\mathrm{N}$ & & 2 \\
\hline Sonchus asper (L.) Hill. & Ñilhue & $\mathrm{Te}$ & I & & 1 \\
\hline Sonchus oleraceus L. & Ñilhue & $\mathrm{Te}$ & I & & 2 \\
\hline $\begin{array}{l}\text { Triptilion spinosum Ruiz et Pav. } \\
\text { - Berberidaceae }\end{array}$ & Siempre viva & $\mathrm{Hc}$ & $\mathrm{N}$ & & 2,4 \\
\hline $\begin{array}{l}\text { Berberis actinacantha Mart. } \\
\text { - } \quad \text { Boraginaceae }\end{array}$ & Michay & $\mathrm{Nf}$ & $\mathrm{N}$ & & $1,2,4$ \\
\hline Amsinckia calycina (Moris) Chater & Ortiguilla & $\mathrm{Te}$ & $\mathrm{N}$ & & 2 \\
\hline Cryptantha aprica (Phil.) Reiche & s.n. & $\mathrm{Te}$ & $\mathrm{N}$ & & 1,4 \\
\hline Plagiobothrys procumbens A.Gray & s.n. & $\mathrm{Te}$ & $\mathrm{N}$ & & 4 \\
\hline
\end{tabular}


Continuación de Anexo 1

\begin{tabular}{|c|c|c|c|c|c|}
\hline Clasificación / Nombre científico & Nombre común & $\mathrm{FV}$ & $\mathrm{OF}$ & $\mathrm{EC}$ & REG \\
\hline \multicolumn{6}{|l|}{ - Brassicaceae } \\
\hline Brassica rapa subsp. campestris (L.) A. R. Clapham & Yuyo & $\mathrm{Te}$ & I & & 2,4 \\
\hline Capsella bursa-pastoris (L.) Medik. & Bolsa de pastor & $\mathrm{Te}$ & I & & 2 \\
\hline Cardamine bonariensis Pers. & s.n. & $\mathrm{Hc}$ & $\mathrm{N}$ & & 2 \\
\hline Lepidium spicatum Desv. & s.n. & $\mathrm{Hc}$ & $\mathrm{N}$ & & 2 \\
\hline Rapistrum rugosum (L.) All. & Falso yuyo & $\mathrm{Te}$ & I & & 2 \\
\hline $\begin{array}{l}\text { Nasturtium officinale R.Br. } \\
\text { - } \quad \text { Cactaceae }\end{array}$ & Berro & $\mathrm{Hc}$ & I & & 2,4 \\
\hline $\begin{array}{l}\text { Eriosyce curvispina (Bertero ex Colla) Katt. } \\
\text { - Caesalpiniaceae }\end{array}$ & s.n. & $\mathrm{C}$ & $\mathrm{N}$ & $\mathrm{V}$ & 2 \\
\hline $\begin{array}{l}\text { Senna candolleana (Vogel) H.S.Irwin et Barneby } \\
\text { - Callitrichaceae }\end{array}$ & Quebracho & $\mathrm{F}$ & $\mathrm{N}$ & & 1,3 \\
\hline $\begin{array}{l}\text { Callitriche palustris L. } \\
\text { - Campanulaceae }\end{array}$ & Huenchecó & $\mathrm{Hi}$ & $\mathrm{N}$ & & 2 \\
\hline $\begin{array}{l}\text { Lobelia excelsa Bonpl. } \\
\text { - Caryophyllaceae }\end{array}$ & Tabaco del diablo & $\mathrm{Nf}$ & $\mathrm{N}$ & & 2,3 \\
\hline Cardionema ramosissimum (Weinm.) A.Nelson et J. F. Macbr. & Dicha & $\mathrm{Hc}$ & $\mathrm{N}$ & & 2 \\
\hline Cerastium fontanum Baumg. subsp. vulgare (Hartm.) Greuter et Burdet & Cerastio & $\mathrm{Te}$ & I & & 1,4 \\
\hline Corrigiola squamosa Hook. et Arn. & Hierba del niño & $\mathrm{Ge}$ & $\mathrm{N}$ & & 2 \\
\hline Sagina apetala Ard. & s.n. & $\mathrm{Te}$ & I & & 2 \\
\hline Silene gallica $\mathrm{L}$. & Calabacillo & $\mathrm{Te}$ & I & & $1,2,4$ \\
\hline Spergularia media (L.) C. Presl ex Griseb. & Tiqui-tiqui & $\mathrm{Te}$ & I & & 2 \\
\hline Stellaria chilensis Pedersen & Quilloi-quilloi & $\mathrm{Hc}$ & $\mathrm{N}$ & & $1,2,4$ \\
\hline $\begin{array}{l}\text { Stellaria media (L.) Cirillo } \\
\text { - Celastraceae }\end{array}$ & Quilloi-quilloi & $\mathrm{Te}$ & I & & 2 \\
\hline $\begin{array}{l}\text { Maytenus boaria Molina } \\
\text { - Chenopodiaceae }\end{array}$ & Maitén & $\mathrm{F}$ & $\mathrm{N}$ & & $1,2,3,4$ \\
\hline $\begin{array}{l}\text { Chenopodium album L. } \\
\text { - Convolvulaceae }\end{array}$ & Quinguilla & $\mathrm{Te}$ & I & & 1 \\
\hline Convolvulus arvensis $\mathrm{L}$. & Corregüela & $\mathrm{Ge}$ & I & & 2,4 \\
\hline Convolvulus chilensis Pers. & Corregüela & $\mathrm{Ge}$ & $\mathrm{N}$ & & 4 \\
\hline $\begin{array}{l}\text { Dichondra sericea } \mathrm{Sw} . \\
\text { - Crassulaceae }\end{array}$ & Oreja de ratón & $\mathrm{Hc}$ & $\mathrm{N}$ & & 2 \\
\hline $\begin{array}{l}\text { Crassula closiana (Gay) Reiche } \\
\text { - Cuscutaceae }\end{array}$ & Flor de piedra & $\mathrm{Te}$ & $\mathrm{N}$ & & 2 \\
\hline $\begin{array}{l}\text { Cuscuta chilensis Ker Gawl. } \\
\text { - Dipsacaceae }\end{array}$ & Cabello de ángel & $\mathrm{Te}$ & $\mathrm{N}$ & & 2 \\
\hline $\begin{array}{l}\text { Dipsacus sativus (L.) Honck. } \\
\text { - } \quad \text { Elaeocarpaceae }\end{array}$ & Carda & $\mathrm{Te}$ & $\mathrm{N}$ & & 1 \\
\hline Aristotelia chilensis (Molina) Stuntz & Maqui & $\mathrm{Nf}$ & $\mathrm{N}$ & & $1,2,3,4$ \\
\hline $\begin{array}{l}\text { Crinodendron patagua Molina } \\
\text { - Escalloniaceae }\end{array}$ & Patagua & $\mathrm{F}$ & $\mathrm{N}$ & & 3 \\
\hline Escallonia pulverulenta (Ruiz et Pav.) Pers. & Corontillo, madroño & $\mathrm{F}$ & $\mathrm{N}$ & & $1,2,3,4$ \\
\hline Escallonia revoluta (Ruiz et Pav.) Pers. & Lun & $\mathrm{Nf}$ & $\mathrm{N}$ & & $1,2,4$ \\
\hline $\begin{array}{l}\text { Escallonia rubra (Ruiz et Pav.) Pers. } \\
\text { - Euphorbiaceae }\end{array}$ & Siete camisas & $\mathrm{Nf}$ & $\mathrm{N}$ & & 3 \\
\hline Colliguaja odorifera Molina & Colliguay & $\mathrm{Nf}$ & $\mathrm{N}$ & & 2,3 \\
\hline Euphorbia klotzschii Oudejans & Pichoguilla & $\mathrm{Te}$ & $\mathrm{N}$ & & 2 \\
\hline Euphorbia peplus L. & Pichoga & $\mathrm{Te}$ & I & & 2 \\
\hline
\end{tabular}


BOSQUE 30(3): 159-179, 2009

Flora y vegetación RN Lago Peñuelas, Chile

Continuación de Anexo 1

Clasificación / Nombre científico

- Fabaceae

Adesmia tenella Hook. et Arn.

Astragalus berteroanus (Moris) Reiche

Lathyrus berteroanus Colla

Lathyrus magellanicus Lam.

Lotus subpinnatus Lag.

Lupinus microcarpus Sims

Medicago arabica (L.) Huds.

Medicago polymorpha $\mathrm{L}$.

Melilotus indicus (L.) All.

Otholobium glandulosum (L.) J.W.Grimes

Robinia pseudoacacia L.

Sophora macrocarpa $\mathrm{Sm}$.

Trifolium dubium Sibth.

Trifolium polymorphum Poir.

Trifolium subterraneum L.

Vicia mucronata $\mathrm{Clos}$

Vicia nigricans Hook. et Arn.

Vicia sativa $\mathrm{L}$.

- Flacourtiaceae

Azara celastrina D. Don

Azara dentata Ruiz et Pav.

Azara petiolaris (D. Don) I.M.Johnst.

- Fumariaceae

Fumaria capreolata L.

Fumaria densiflora DC.

- Geraniaceae

Erodium botrys (Cav.) Bertol.

Erodium cicutarium (L.) L'Hér. ex Aiton

Erodium malacoides (L.) L'Hér. ex Aiton

Erodium moschatum (L.) L'Hér. ex Aiton

Geranium berteroanum Colla

Geranium core-core Steud.

- Grossulariaceae

Ribes punctatum Ruiz et Pav.

- Gunneraceae

Gunnera tinctoria (Molina) Mirb.

- Haloragaceae

Myriophyllum sp.

- Hydrophyllaceae

Phacelia secunda J.F.Gmel.

- Lamiaceae

Lamiun amplexicaule L.

Marrubium vulgare $\mathrm{L}$.

Mentha pulegium L.

Satureja gilliesii (Graham) Briq.

Stachys albicaulis Lindl.

Stachys grandidentata Lindl.

Teucrium bicolor Sm.
Nombre común

FV

$\mathrm{OF}$

EC

REG.

Trebillo

Yerba loca

Clarincillo, arvejilla

Clarincillo, arvejilla

Porotillo

Arvejilla

Hualputra

Hualputra

Trevillo

Culén

Falsa acacia

Pelu

Trebillo

Trébol

Trébol subterráneo

Arvejilla

Arvejilla

Arvejilla, clarincillo

Lilén, corcolén

Corcolén

Corcolén

Flor de la culebra

Flor de la culebra

Alfilerillo

Alfilerillo

Alfilerillo

Alfilerillo

Core-core

Core-core

Zarzaparrilla

Nalca

Pinito de agua

Té de burro

Gallito

Toronjil cuyano

Poleo

Oreganillo

s.n.

Hierba santa

Oreganillo

Te $\mathrm{N} \quad 2,4$

Te $\mathrm{N} 2$

Hc N 2

Hc N 2

Te $\mathrm{N} \quad 2$

Te $\mathrm{N} \quad 1,2$

Te I 2

Te I 1,2

Te $\mathrm{I} \quad 2$

F N $1,3,4$

F I 1

$\mathrm{Nf} \mathrm{N}$

$\begin{array}{lll}\mathrm{NI} & \mathrm{N}\end{array}$

Te I 1

Hc N 2

Ge $\mathrm{I} \quad 2$

Te $N \quad 2$

Te N 2

$\begin{array}{ccc}\mathrm{Te} & \mathrm{N} & 2 \\ \mathrm{Te} & \mathrm{I} & 1\end{array}$

F

$\begin{array}{lll}\mathrm{F} & \mathrm{N} & 2,3\end{array}$

$\mathrm{Nf} \quad \mathrm{N} \quad 1,2$

Nf $\mathrm{N} \quad 4$

Te I 1,5

Te $\quad$ I $2,4,5$

Te 2

Te $\quad 2$

Te I 2

Te I $1,2,4$

$\mathrm{Hc} \quad \mathrm{N} 2,4$

Hc $\mathrm{N} \quad 1,2$

$\mathrm{Nf} \quad \mathrm{N} \quad 2,4$

Ge $\quad \mathrm{N} \quad 1$

Ni $\quad 3$

Hc $\quad \mathrm{N}, 4$

Te I 2, 4

Hc I 1,2

Hc I 1

Nf $\mathrm{N} \quad 1,2,3,4$

$\mathrm{Hc} \quad \mathrm{N} \quad 4$

$\mathrm{Hc} \quad \mathrm{N} \quad 1,2$

$\mathrm{Nf} \quad \mathrm{N} \quad 1,2$

Continúa 
Continuación de Anexo 1

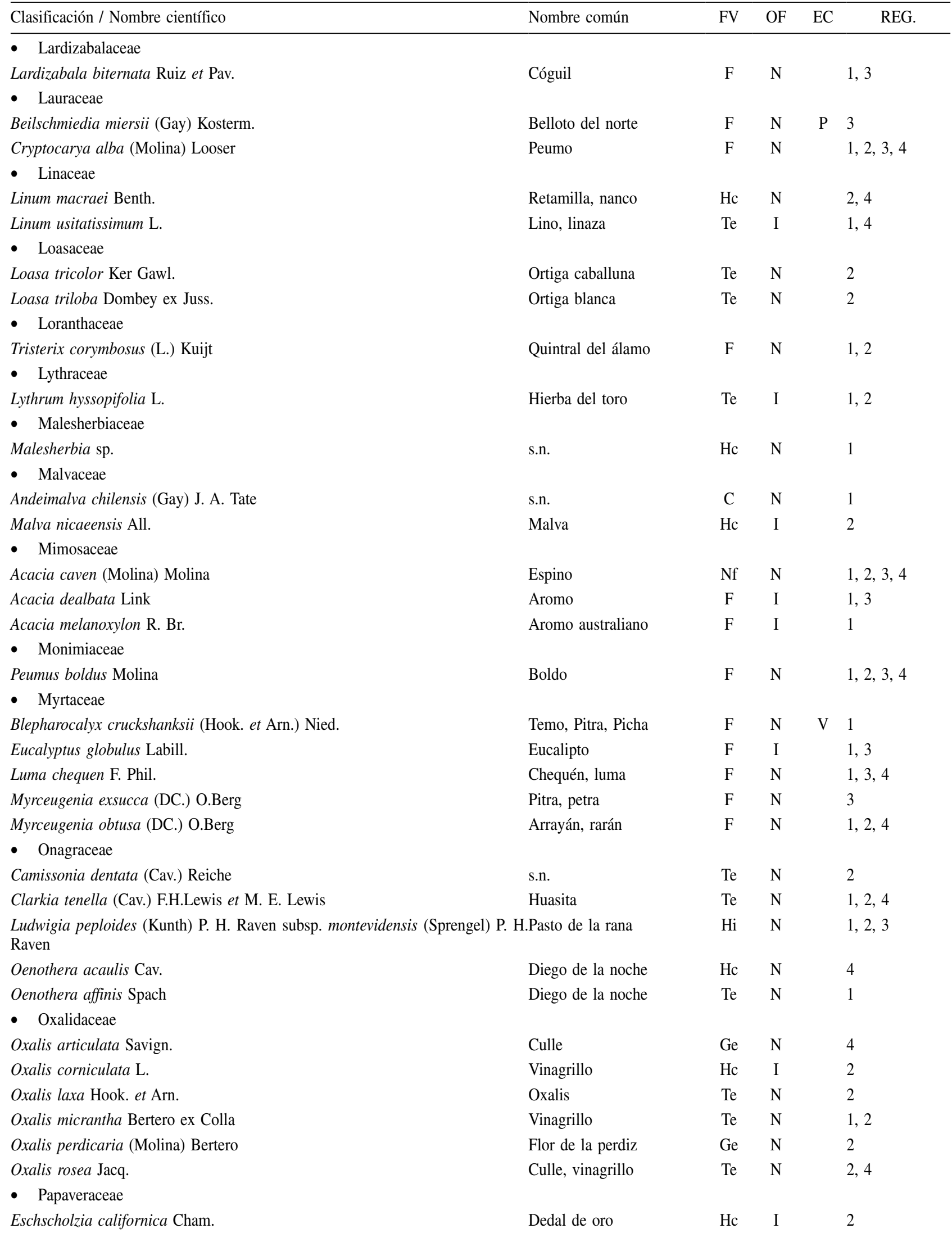


Continuación de Anexo 1

Clasificación / Nombre científico

- Plantaginaceae

Plantago lanceolata L.

Plantago hispidula Ruiz et Pav.

- Plumbaginaceae

Armeria maritima (Mill.) Willd.

- Polygalaceae

Monnina sp.

- Polygonaceae

Muehlenbeckia hastulata (Sm.) I.M.Johnst.

Polygonum hydropiperoides Michx.

Polygonum persicaria $\mathrm{L}$.

Rumex acetosella $\mathrm{L}$.

Rumex conglomeratus Murray

Rumex crispus $\mathrm{L}$.

Rumex pulcher $\mathrm{L}$.

Calandrinia nitida (Ruiz et Pav.) DC.

Calandrinia compressa Schrad. ex DC.

- Primulaceae

Anagallis alternifolia Cav.

Anagallis arvensis $\mathrm{L}$.

- Ranunculaceae

Anemone decapetala Ard.

Ranunculus chilensis DC.

Ranunculus muricatus L.

- Rhamnaceae

Retanilla trinervia (Guillies et Hook.) Hook. et Arn.

- Rosaceae

Acaena pinnatifida Ruiz et Pav.

Margyricarpus pinnatus (Lam.) Kuntze

Quillaja saponaria Molina

Rosa moschata Herrm.

Rosa rubiginosa $\mathrm{L}$.

Rubus ulmifolius Schott

- Rubiaceae

Galium aparine L.

Galium hypocarpium (L.) Endl. ex Griseb.

Galium trichocarpum DC.

- Salicaceae

Populus deltoides Bartram ex Marshall

Populus nigra $\mathrm{L}$.

Salix babylonica $\mathrm{L}$

- Santalaceae

Quinchamalium chilense Molina

- Scrophulariaceae

Alonsoa meridionalis (L. f.) Kuntze

Bartsia trixago $\mathrm{L}$.

Calceolaria ascendens Lindl.

Calceolaria corymbosa Ruiz et Pav.

Calceolaria petioalaris Cav.
Nombre común

Siete venas

s.n.

s.n.

s.n.

Voqui negro

Duraznillo

Duraznillo

Vinagrillo, romacilla

Romaza

Romaza

Romaza

s.n.

s.n.

s.n.

Pimpinela azul

Centella

Ranúnculo

Centella, huante

Trevo, tebo

Cadillo, pimpinela

Perlilla

Quillay

Mosqueta, coral

Mosqueta, coral

Zarzamora, mora

Lengua de gato

Relbún

s.n.

Álamo americano

Álamo negro

Sauce llorón

Quinchamalí

s.n.

Belardia

s.n.

Arguenita del cerro

Capachito, topa-topa

FV OF EC

REG.

$1,2,3,4$

Hc I

2

Te N 2

$\mathrm{Hc} \quad \mathrm{N}$

2

Nf N

4

$1,2,3,4$

$1,3,4$

1,2

1

4

1,2

2

2

Te $\mathrm{N} \quad 2$

Te $\quad 2$

Te I $\quad 1,4$

1,4

$\begin{array}{lll} & \mathrm{N} & 2\end{array}$

Te $\mathrm{N} \quad 4$

Hc I 2, 4

$\mathrm{Nf} \quad \mathrm{N} \quad 1,2,3,4$

$\begin{array}{lll}\mathrm{H} & \mathrm{N} & \end{array}$

C $\mathrm{N} 2$

F N

$1,2,3,4$

Nf I 3

$\mathrm{Nf} \quad \mathrm{I} \quad 1$

Nf I $\quad 1,2,3,4$

Te $\quad$ I 2

Te $\mathrm{N} \quad 2$

Hc $\quad \mathrm{N} \quad 4$

F I 1

F I 1,2

F I 1

$\mathrm{Hc} \quad \mathrm{N}, 4$

$\mathrm{H} \quad 1,2,3,4$

Te I 1,4

$\mathrm{Hc} \quad \mathrm{N} \quad 2,4$

$\mathrm{Hc} \quad \mathrm{N} \quad 2$

$\mathrm{Hc} \quad \mathrm{N} \quad 4$

Continúa 
Continuación de Anexo 1

\begin{tabular}{|c|c|c|c|c|c|}
\hline Clasificación / Nombre científico & Nombre común & $\mathrm{FV}$ & $\mathrm{OF}$ & $\mathrm{EC}$ & REG. \\
\hline Calceolaria sp. & Topa-topa & $\mathrm{Hc}$ & $\mathrm{N}$ & & 4 \\
\hline Calceolaria thyrsiflora Graham & Hierba dulce & $\mathrm{Hc}$ & $\mathrm{N}$ & & 1 \\
\hline Verbascum virgatum Stokes & Raspa la choica & $\mathrm{Te}$ & I & & 1,4 \\
\hline Veronica anagallis-aquatica $\mathrm{L}$. & No me olvides & $\mathrm{Hi}$ & I & & 4 \\
\hline $\begin{array}{l}\text { Veronica arvensis L. } \\
\text { - Solanaceae }\end{array}$ & Verónica & $\mathrm{Te}$ & I & & 2 \\
\hline Cestrum parqui L'Hér. & Palqui & $\mathrm{Nf}$ & $\mathrm{N}$ & & $1,2,3$ \\
\hline Fabiana imbricata Ruiz et Pav. & Pichi & $\mathrm{Nf}$ & $\mathrm{N}$ & & 3 \\
\hline Lycium chilense Miers ex Bertero & Coralillo & $\mathrm{Nf}$ & $\mathrm{N}$ & & 2 \\
\hline Nicotiana acuminata (Graham) Hook. & Tabaco del campo & $\mathrm{Te}$ & $\mathrm{N}$ & & 1 \\
\hline Schizanthus litoralis Phil. & Pajarito, mariposita & $\mathrm{Te}$ & $\mathrm{N}$ & & 2 \\
\hline $\begin{array}{l}\text { Solanum furcatum Dunal ex Poir. } \\
\text { - Tropaeolaceae }\end{array}$ & Tomatillo & $\mathrm{C}$ & $\mathrm{N}$ & & $1,2,4$ \\
\hline $\begin{array}{l}\text { Tropaeolum tricolor Sweet } \\
\text { - Urticaceae }\end{array}$ & Soldadillo, relicario & $\mathrm{Ge}$ & $\mathrm{N}$ & & 2,4 \\
\hline $\begin{array}{l}\text { Urtica urens } \mathrm{L} . \\
\text { - Valerianaceae }\end{array}$ & Ortiga & $\mathrm{Te}$ & I & & 2 \\
\hline $\begin{array}{l}\text { Valeriana verticillata } \text { Clos } \\
\text { - Verbenaceae }\end{array}$ & Valeriana & $\mathrm{Hc}$ & $\mathrm{N}$ & & 4 \\
\hline Glandularia laciniata (L.) Schnack et Covas & Hierba del incornio & $\mathrm{Hc}$ & $\mathrm{N}$ & & $1,2,4$ \\
\hline Phyla canescens (Kunth) Greene & Hierba de la Virgen & $\mathrm{Hc}$ & $\mathrm{N}$ & & 1 \\
\hline Rhaphithamnus spinosus (Juss.) Moldenke & Arrayán macho & $\mathrm{Nf}$ & $\mathrm{N}$ & & 1,3 \\
\hline $\begin{array}{l}\text { Verbena bonariensis L. } \\
\text { - Violaceae }\end{array}$ & Verbena & $\mathrm{Hc}$ & $\mathrm{N}$ & & 1 \\
\hline $\begin{array}{l}\text { Viola } \text { sp. } \\
\text { - Vitaceae }\end{array}$ & Violeta del campo & $\mathrm{Te}$ & $\mathrm{N}$ & & 2 \\
\hline $\begin{array}{l}\text { Cissus striata Ruiz et Pav. } \\
\text { - Winteraceae }\end{array}$ & Pilpilvoqui & $\mathrm{F}$ & $\mathrm{N}$ & & 1,3 \\
\hline $\begin{array}{l}\text { Drimys winteri J. R. Forst. et G. Forst. var. chilensis (DC.) A. Gray } \\
\text { B) Monocotyledoneae (Liliopsida) } \\
\text { - Alismataceae }\end{array}$ & Canelo & $\mathrm{F}$ & $\mathrm{N}$ & & 1,3 \\
\hline $\begin{array}{l}\text { Alisma plantago-aquatica } \mathrm{L} \text {. } \\
\text { - Alliaceae }\end{array}$ & Llantén de agua & $\mathrm{Hi}$ & I & & 4 \\
\hline Gilliesia graminea Lindl. & Junquillo & $\mathrm{Ge}$ & $\mathrm{N}$ & & 2,4 \\
\hline Leucocoryne ixioides (Sims) Lindl. & Huilli & $\mathrm{Ge}$ & $\mathrm{N}$ & $\mathrm{V}$ & $1,2,4$ \\
\hline Leucocoryne violacescens Phil. & s.n. & $\mathrm{Ge}$ & $\mathrm{N}$ & $\mathrm{V}$ & 1 \\
\hline Nothoscordum gramineum (Sims) Beauverd & Huilli de perro & $\mathrm{Ge}$ & $\mathrm{N}$ & & 4 \\
\hline $\begin{array}{l}\text { Tristagma bivalve (Lindl.) Traub } \\
\text { - Alstroemeriaceae }\end{array}$ & s.n & $\mathrm{Ge}$ & $\mathrm{N}$ & & 2 \\
\hline Alstroemeria angustifolia Herb. & Lirio del campo & $\mathrm{Ge}$ & $\mathrm{N}$ & & 1,4 \\
\hline Alstroemeria hookeri Lodd. ssp. recumbens & Lirio del campo & $\mathrm{Ge}$ & $\mathrm{N}$ & $\mathrm{V}$ & 2 \\
\hline $\begin{array}{l}\text { Alstroemeria pulchra Sims ssp. pulchra } \\
\text { - Amarillidaceae }\end{array}$ & Lirio del campo & $\mathrm{Ge}$ & $\mathrm{N}$ & & $1,2,3,4$ \\
\hline Hippeastrum bicolor (Ruiz et Pav.) Baker & Añañuca de fuego & $\mathrm{Ge}$ & $\mathrm{N}$ & $\mathrm{V}$ & 2,4 \\
\hline $\begin{array}{l}\text { Rhodophiala advena (Ker Gawl.) Traub. } \\
\text { - Anthericaceae }\end{array}$ & Añañuca & $\mathrm{Ge}$ & $\mathrm{N}$ & & 1,2 \\
\hline Pasithea caerulea (Ruiz et Pav.) D.Don & Azulillo & $\mathrm{Ge}$ & $\mathrm{N}$ & & 1,4 \\
\hline Trichopetalum plumosum (Ruiz et Pav.) J. F. Macbr. & Flor de la plumilla & $\mathrm{Ge}$ & $\mathrm{N}$ & & 4 \\
\hline
\end{tabular}


Continuación de Anexo 1

Clasificación / Nombre científico

- Bromeliaceae

Puya chilensis Molina

- Cyperaceae

Carex excelsa Poepp. ex Kunth

Carex setifolia Kunze ex Kunth

Carex sp.

Cyperus eragrostis Lam. var. compactus (E. Desv.) Kük.

Cyperus eragrostis Lam. var. eragrostis

Eleocharis radicans (Poir.) Kunth

Eleocharis pachycarpa E. Desv.

Isolepis cernua (Vahl) Roem. et Schult.

Schoenoplectus californicus (C. A. Mey.) Soják

Scirpus asper J. Presl et C.Presl

Uncinia trichocarpa C. A. Mey.

- Dioscoreaceae

Dioscorea saxatilis Poepp.

- Hyacinthaceae

Oziroe biflora (Ruiz et Pav.) Speta

- Hydrocharitaceae

Elodea potamogeton (Bertero) Espinosa

- Iridaceae

Calydorea xiphioides (Poepp.) Espinosa

Herbertia lahue (Molina) Goldblat

Sisyrinchium arenarium Poepp.

Sisyrinchium chilense Hook.

Sisyrinchium cuspidatum Poepp.

Olsynium junceum (E. Mey. ex C. Presl) Goldblatt

Solenomelus pedunculatus (Guillies ex Hook.) Hochr.

- Juncaceae

Juncus acutus L.

Juncus bufonius L.

Juncus cyperoides Laharpe

Juncus imbricatus Laharpe

Juncus pallescens Lam.

Juncus stipulatus Nees et Meyen

Luzula racemosa Desv.

- Lemnaceae

Lemna valdiviana Phil.

- Orchidaceae

Brachystele unilateris (Poir.) Schltr.

Chloraea barbata Lindl.

Chloraea bletioides Lindl.

Chloraea chrysantha Poepp.

Chloraea cylindrostachya Poepp.

Chloraea disoides Lindl.

Chloraea galeata Lindl.

Chloraea heteroglossa Rchb. f.

Chloraea virescens (Willd.) Lindl.

Chloraea multiflora Lindl.
Nombre común

Chagual, Cardón

Cortadera

Cortadera

s.n.

Cortadera

Cortadera

s.n

Rime

s.n

Totora, estoquilla

s.n

Clin clin

Papa cimarrona

Cebolleta

Luchecillo

s.n

Lahue

Huilmo, ñuño

Huilmo, ñuño

Huilmo, ñuño

Huilmo, ñuño

Maicillo

Junquillo

Junquillo

s.n.

Junquillo

Junco

Junco

s.n

Lenteja de agua

Orquídea

Orquídea

Orquídea

Orquídea

Orquídea

Orquídea

Orquídea

Orquídea

Orquídea

Orquídea

FV OF EC REG.

$\mathrm{Hc} \quad \mathrm{N} \quad \mathrm{V} \quad 1,2,3,4$

Hc $\quad \mathrm{N} \quad 1$

$\mathrm{Hc} \quad \mathrm{N} \quad 4$

$\mathrm{Hc} \quad \mathrm{N} \quad 1$

Ge $\quad \mathrm{N} \quad 1,4$

Ge $\quad \mathrm{N} \quad 1,2,4$

$\mathrm{Hc} \quad \mathrm{N} \quad 1,2$

$\mathrm{Hc} \quad \mathrm{N} \quad 3$

Ge $\quad \mathrm{N} \quad 2$

Ge $\mathrm{N} \quad 1,2$

Ge $\mathrm{N} \quad 4$

Hc $\quad \mathrm{N} \quad 1$

Ge $\quad \mathrm{N} \quad 1,2,4$

Ge $\quad \mathrm{N} \quad 4,2$

Hi N 3

$\begin{array}{llll}\mathrm{Ge} & \mathrm{N} & \mathrm{P} & 4\end{array}$

Ge $\quad \mathrm{N} \quad \mathrm{V} \quad 4$

Ge $\mathrm{N} \quad 4$

Ge $\mathrm{N} \quad 4$

Ge $\mathrm{N} 2$

Ge $\quad \mathrm{N} \quad 1,2,4$

Ge $\quad \mathrm{N} \quad 4$

N 1,4

$\begin{array}{lll}\mathrm{Te} & \mathrm{I} & 1,2\end{array}$

$\mathrm{Hc} \quad \mathrm{N} \quad 1,2$

Ge $\quad \mathrm{N} \quad 1$

$\mathrm{Hc} \quad \mathrm{N} \quad 1$

$\mathrm{Hc} \quad \mathrm{N} \quad 1,4$

$\mathrm{Hc} \mathrm{N} \quad 1,2$

Hi N 2

Ge $\quad \mathrm{N} \quad 6$

Ge $\quad \mathrm{N} \quad 6$

$\mathrm{Ge} \quad \mathrm{N}$

Ge N

$1,2,4,6$

$1,4,6$

$\mathrm{Ge} \quad \mathrm{N} \quad 4,6$

Ge N V 4,6

Ge $\mathrm{N} \quad 6$

Ge $\quad \mathrm{N} \quad \mathrm{V} 6$

Ge $\quad \mathrm{N} \quad 6$

Ge $\quad \mathrm{N} \quad 4,6$ 
Continuación de Anexo 1

\begin{tabular}{|c|c|c|c|c|c|}
\hline Clasificación / Nombre científico & Nombre común & $\mathrm{FV}$ & $\mathrm{OF}$ & $\mathrm{EC}$ & REG. \\
\hline Bipinnula mystacina Lindl. & Orquídea & $\mathrm{Ge}$ & $\mathrm{N}$ & & 2 \\
\hline Gavilea venosa (Lam.) Garay et Ormd. & Orquídea & $\mathrm{Ge}$ & $\mathrm{N}$ & & 4,6 \\
\hline $\begin{array}{l}\text { Gavilea leucantha Poepp. et Endl. } \\
\text { - Poaceae }\end{array}$ & Orquídea & $\mathrm{Ge}$ & $\mathrm{N}$ & & 6 \\
\hline Agrostis capillaris $\mathrm{L}$. & Chépica & $\mathrm{Hc}$ & I & & 1,2 \\
\hline Aira caryophyllea $\mathrm{L}$. & s.n. & $\mathrm{Te}$ & I & & 1 \\
\hline Avena barbata Pott ex Link & Teatina & $\mathrm{Te}$ & I & & $1,2,4$ \\
\hline Briza maxima $\mathrm{L}$ & Tembladera & $\mathrm{Te}$ & I & & 1 \\
\hline Briza minor $\mathrm{L}$. & Pasto perdiz & $\mathrm{Te}$ & I & & $1,2,4$ \\
\hline Bromus catharticus Vahl & Pasto lanco & $\mathrm{Hc}$ & $\mathrm{N}$ & & 2,4 \\
\hline Bromus hordeaceus L. & Bromo, cebadilla & $\mathrm{Te}$ & $\mathrm{I}$ & & 1,4 \\
\hline Chascolytrum subaristatum (Lam.) Desv. & Tembladera & $\mathrm{Te}$ & $\mathrm{N}$ & & $1,2,4$ \\
\hline Chusquea cumingii Nees & Coligüe, quila & $\mathrm{Nf}$ & $\mathrm{N}$ & & $1,2,3$ \\
\hline Hordeum chilense Roem. et Schult. & Cebadilla & $\mathrm{Hc}$ & $\mathrm{N}$ & & 4 \\
\hline Hordeum murinum L. & Cebadilla & $\mathrm{Te}$ & I & & 2,4 \\
\hline Cynodon dactylon (L.) Pers. & Pasto bermuda & $\mathrm{Hc}$ & I & & 1 \\
\hline Cynosurus echinatus L. & Cola de zorro & $\mathrm{Te}$ & I & & 2,4 \\
\hline Distichlis spicata (L.) Greene & Pasto salado & $\mathrm{Ge}$ & $\mathrm{N}$ & & 1 \\
\hline Echinochloa colonum (L.) Link & Hualcacho & $\mathrm{Te}$ & $\mathrm{N}$ & & 1 \\
\hline Eragrostis virescens J. Presl. & S.n & $\mathrm{Te}$ & $\mathrm{N}$ & & 1,4 \\
\hline Festuca sp. & Coirón & $\mathrm{Hc}$ & $\mathrm{N}$ & & 1 \\
\hline Gastridium phleoides (Nees et Meyen) C. E. Hubb. & s.n & $\mathrm{Te}$ & I & & 1,4 \\
\hline Lolium multiflorum Lam. & Ballica italiana & $\mathrm{Te}$ & I & & 2,4 \\
\hline Lolium perenne $\mathrm{L}$. & Ballica inglesa & $\mathrm{Ge}$ & $\mathrm{I}$ & & 1,4 \\
\hline Melica longiflora Steud. & s.n & $\mathrm{Te}$ & $\mathrm{N}$ & & 2,4 \\
\hline Melica violacea $\mathrm{Cav}$ & S.n & $\mathrm{Te}$ & $\mathrm{N}$ & & $1,2,4$ \\
\hline Nassella chilensis (Trin.) E.Desv. & Coironcillo & $\mathrm{Hc}$ & $\mathrm{N}$ & & 2,4 \\
\hline Nassella gigantea (Steud.) M.Muñoz & s.n & $\mathrm{Hc}$ & $\mathrm{N}$ & & 4 \\
\hline Nassella manicata (Desv.) Barkworth & S.n & $\mathrm{Hc}$ & $\mathrm{N}$ & & 4 \\
\hline Paspalum dilatatum Poir. & Chépica gigante & $\mathrm{Ge}$ & $\mathrm{N}$ & & 1 \\
\hline Paspalum vaginatum $\mathrm{Sw}$. & s.n & $\mathrm{Ge}$ & $\mathrm{N}$ & & 2 \\
\hline Phleum pratense $\mathrm{L}$. & S.n & $\mathrm{Hc}$ & I & & 4 \\
\hline Piptochaetium montevidense (Spreng.) Parodi & s.n & $\mathrm{Hc}$ & $\mathrm{N}$ & & 2 \\
\hline Piptochaetium panicoides (Lam.) Desv. & s.n & $\mathrm{Hc}$ & $\mathrm{N}$ & & 2 \\
\hline Piptochaetium stipoides (Trin. et Rupr.) Hackel & S.n & $\mathrm{Te}$ & $\mathrm{N}$ & & 4 \\
\hline Pоа аппиа $\mathrm{L}$. & Pasto piojillo & $\mathrm{Te}$ & I & & 1 \\
\hline Rostraria cristata (L.) Tzvelev & s.n & $\mathrm{Te}$ & I & & 2,4 \\
\hline Setaria parviflora (Poir.) Kerguélen & s.n & $\mathrm{Hc}$ & $\mathrm{N}$ & & 2 \\
\hline Stipa sp. & Flechilla & $\mathrm{Hc}$ & $\mathrm{N}$ & & 1,2 \\
\hline Vulpia bromoides (L.) Gray & Pasto sedilla & $\mathrm{Te}$ & I & & 1,2 \\
\hline $\begin{array}{l}\text { Vulpia myuros (L.) C.C.Gmel. } \\
\text { - } \quad \text { Potamogetonaceae }\end{array}$ & Pasto largo & $\mathrm{Te}$ & I & & $1,2,4$ \\
\hline $\begin{array}{l}\text { Potamogeton pusillus L. } \\
\text { - Tecophilaeaceae }\end{array}$ & Huiro & $\mathrm{Hi}$ & $\mathrm{N}$ & & 1,3 \\
\hline Conanthera bifolia Ruiz et Pav. & Pajarito del campo & $\mathrm{Ge}$ & $\mathrm{N}$ & & 4 \\
\hline Conanthera campanulata (D. Don) Lindl. & Pajarito del campo & $\mathrm{Ge}$ & $\mathrm{N}$ & & 1,2 \\
\hline $\begin{array}{l}\text { Conanthera trimaculata (D. Don) Fr.Meigen } \\
\text { - Typhaceae }\end{array}$ & Pajarito del campo & $\mathrm{Ge}$ & $\mathrm{N}$ & V & 1,4 \\
\hline Typha angustifolia $\mathrm{L}$. & Vatro & $\mathrm{Ge}$ & $\mathrm{N}$ & & 1 \\
\hline
\end{tabular}

